\title{
Die „Kultur-Hygiene“ des Eduard Reich - Ein Rückblick zur 100. Wiederkehr seines Todesjahres
}

\author{
Heinz Flamm
}

Eingegangen: 9. März 2018 / Angenommen: 12. August 2018 / Online publiziert: 4. September 2018

(c) Der/die Autor(en) 2018

Zusammenfassung Entgegen der in der zweiten Hälfte des 19. Jahrhunderts steigenden Bedeutung der sich entwickelnden Bakteriologie und der Kenntnis umweltbedingter chemischer und physikalischer Noxen sieht Eduard Reich als Hauptgrund für die Entscheidung zwischen Gesundheit und Krankheit die Funktionen der „Seele“. Davon seien für das normale Leben von Bedeutung: „Tugend“ (das ist Gemeingeist, Pflichterfüllung, Selbsterkenntnis), Vernunft zum Erkennen der Zusammenhänge, Sittlichkeit sowie Nächstenliebe und „Barmherzigkeit“ (soziales Verhalten). Das Ziel der Hygiene sei somit, das Gute $\mathrm{zu}$ fördern und das Böse $\mathrm{zu}$ verhindern. Sie bewirkt damit Gesundheit, Tugend und Glück, einerseits für den ganzen Menschen oder einzelne seiner Organe andererseits auch für die Gesellschaft. Das Endziel der Hygiene ist für Reich aber nicht bloß die Erhöhung der Leistungsfähigkeit der Bevölkerung für irgendwelche ökonomische oder militärische Zwecke, sondern auch die Gesunderhaltung der Seele.

Entsprechend den Aufgaben und Zielen der Hygiene teilt Reich diese in vier Gebiete.

Die Moralische Hygiene will mit Hilfe von Erziehung, Unterricht und Religion die durch das Gehirn geleiteten Handlungen, Gemütszustände und Leidenschaften für den Normalzustand regulieren.

Die Soziale Hygiene bemüht sich um das Wohl der ganzen Bevölkerung. Das betraf zu Reichs Zeit besonders Fragen der Arbeit, als deren Grundpfeiler er Moral, Hygiene und Ökonomie erkannt und bearbeitet

Univ.-Prof. Dr. Dr. Heinz Flamm ML ist ehem. Vorstand des Klinischen Instituts für Hygiene der Universität Wien. Die hier angegebene Adresse ist die Privatanschrift.

Univ.-Prof. Dr. Dr. H. Flamm, ML ( $ه)$

Martinstraße 7, 3400 Klosterneuburg, Österreich office.martinstr@aon.at hat, und damals wie heute auch die Folgen von Migrationen.

Die Diätetische Hygiene, wohl die historisch älteste Aufgabe der Gesundheitserhaltung, kümmert sich um die leiblichen Bedürfnisse des Menschen und den Gebrauch seiner Organe. Hierfür sind Vernunft und Vorsicht, Übung und Mäßigung, also Moral und körperliche Ertüchtigung Voraussetzung. Das Ziel ist nicht nur die Erhaltung der Gesundheit, sondern auch die Erreichung eines hohen Alters in Gesundheit.

Die Polizeiliche Hygiene (Gesundheitspolizei) hat durch Erlass und Sicherung der Einhaltung von Maßnahmen für die Gesundheit des Volkes zu sorgen. Sie muss mit Barmherzigkeit und Menschenfreundlichkeit vorgehen und, da das Elend ihr ärgster Gegner ist, die falschen Ansichten einer gefühllosen Ökonomie paralysieren. Die Aufgaben der Gesundheitspolizei der einzelnen Verwaltungsregionen sollen Wohlfahrtsräte mit je einem gesetzgebenden und einem vollziehenden Teil übernehmen, und zwar je ein Wohlfahrtsrat für Gesundheit, für Erziehung und für Sicherheit.

Reichs Auffassung von der Hygiene als Ergebnis der Anwendung von Moral und Nächstenliebe im Leben sowohl jedes Einzelnen als auch der ganzen Gesellschaft rechtfertigt deren wieder vergessene Bezeichnung als „Kultur-Hygiene“.

Schlüsselwörter Gesundheit · Seele · Tugend · Barmherzigkeit · Moral · Ethik

\section{Eduard Reich's "Hygiene of \\ Culture" - A retrospective on the occasion of the $100^{\text {th }}$ anniversary of his death}

Summary In contrary to the developing significance of the bacteriology and the chemical and physical noxious influences of the environment for Eduard Reich the main cause of decision between health and 
illness are the functions of the "soul". Components of this as important for the normal life are virtue (i.e. solidarity, performance of duty, self-knowledge), good sense to realize correlations, morality, charity and mercifulness (social behaviour). The goal of the hygiene therefore is to promote the good and to hinder the evil, as well for a complete person or only one of his organs as for the whole society. The final aim is not only the improvement of the common efficiency for any economic or military purposes but also the preservation of health of the soul.

In accordance to these duties and aims Reich divides the hygiene into four parts.

The "Moral Hygiene" regulates the activities directed by the brain supported by education, training and religion.

The "Social Hygiene" cares for the wealth of the whole population. In Reich's time, it applied in particular to the labour, the basis for it are moral, hygiene and economy, but migration too played a great role.

The "Dietetic Hygiene"-the oldest task of the preservation of health-cares for the human somatic necessities and the use of the organs. Prerequisite for this are bodily exercise and moderation, hence moral and physical training. The aim is not only preserving of the health but also gaining high age in health.

The "Policed Hygiene" (Sanitary Police) has to care for the observance of reassurances for the public health. It must be done with charity and kindness and has to paralyse a heartless economy as poverty is the worst enemy of health. The duties of the sanitary police in the various districts should be fulfilled by boards of health, of education and of public safety. Each of these boards consists of a legislative and an executive part.

Reich's concept of the hygiene as result of the application of moral and charity as well in the lifetime of each individual as of the whole society justifies the already forgotten terminus "Hygiene of Culture".

Keywords Health - Soul · Virtue - Charity - Moral · Ethics

Die Hygiene, wie wir sie lernen, lehren und anwenden, beschäftigt sich im Prinzip mit den von außen auf den Menschen einwirkenden Krankheitsursachen. Eine andere Sicht beschrieb jedoch Eduard Reich 1870 für die Gesellschaft der Ärzte in Wien. Für ihn war die „Hygieine“ [sic! von Hygieia] „die Gesamtheit jener Lehren, deren Anwendung die Erhaltung der individuellen und sozialen Gesundheit, der Sittlichkeit, der Zerstörung der Krankheitsursachen und die Veredelung des Menschen in physischer wie moralischer Beziehung abzweckt. Es umfasst also der Begriff Hygieine weit mehr, als man ehedem unter Diätetik und medizinischer Polizei verstanden hat. Die Hygieine hat es mit dem ganzen Menschen, wie er als Individuum, in Familie und Gesellschaft sich zeigt, zu tun, mit seinen Zuständen und Verhältnissen; sie umfasst demnach die ganze physische und moralische Welt und kommuniziert mit allen Wissenschaften, deren Gegenstand die Betrachtung des Menschen und der diesen umgebenden Welt ist“ [1]. Und „wenn es eine Seele gibt“, so „muss Hygieine des Leibes zuletzt übereinkommen mit Hygieine der Seele, beide müssen einander bedingen, durchdringen, gegenseitig voraussetzen, im Wesen die nämlichen sein und nur in der Form der Anwendung verschieden“ [2].

Für diesen weit gefassten Begriff der Hygiene wurde in den 1920er-Jahren der Begriff „Kulturhygiene“ gefunden und sogar für Kulturhygienische Ausstellungen [3] offiziell verwendet. Reich selbst wurde 1936 in einem kurzen Gedenkartikel zum 100. Geburtstag [4] zu Recht als „Kulturhygieniker“ bezeichnet. Der Begriff der Kulturhygiene ging danach wohl deshalb verloren, weil das 1931 gegründete Eduard-Reich-Archiv des Deutschen Hygiene-Museums im Zweiten Weltkrieg durch Luftangriffe auf Dresden vernichtet worden ist.

Eduard Maria Anton Johann Reich wurde am 6. März 1836 in Sternberg in Mähren [heute: Šternberk, $\check{C} R]$ als Spross österreichischer handwerklicher Familien geboren. In späteren Jahren schrieb er einmal, er „gehöre nur geistig der deutschen Nation an“, sein Blut sei „das der slawischen Rasse, mit romanischen Elementen“ [5]. Reich wurde nach dem Ritus der römischen Kirche getauft, obwohl die Familien nicht besonders katholisch waren, was Letzteres sich offenbar auf das spätere Leben Eduard Reichs in seiner Haltung zum Klerus auswirken sollte. Im folgenden Jahr zog die Familie nach Olmütz [heute: Olomouz, $\check{C} R$ ]. Nach Besuch der Trivial- und der Normal-Schule und des k. k. akademischen Gymnasiums trat Reich in die Stabsschule eines Artillerie-Regiments ein. Neben dem dort mit Eifer und Fleiß gefolgten militärischen Unterricht betrieb er privat chemische Studien und Experimente, noch bevor er „Vorträge über diese herrliche Wissenschaft gehört hatte“. Das strenge „Korporal-Stocks-Regiment“ veranlasste ihn, der „Soldaterei“ den Rücken zu kehren und sich auf das Studium der Natur-Wissenschaften, der Chemie und gesamten Medizin zu werfen. In den folgenden zehn Semestern studierte er die Natur- und Heilkunde, „und zwar in $* * * * *, * * * * * *$ und ${ }^{* * * * *}$. Diese Anonymisierung wird durch eine handschriftliche Eintragung im Exemplar der Autobiographie in der Forschungsbibliothek Gotha als Brünn, Olmütz und Jena aufgelöst, wofür auch die Anzahlen der Sterne stimmen. Schon im dritten Semester hielt Reich als Assistent des chemischen Universitäts-Laboratoriums Vorlesungen über „reine und medizinisch-pharmazeutische Chemie, so wie über Physik in ihrer Anwendung auf Medizin“. Darüber schrieb er in seiner Autobiographie stolz: „der achtzehnjährige Jüngling lehrte dreißig- und vierzigjährigen Männern die vielgeliebte Wissenschaft der Chemie und die Physik“ [5-8]. 


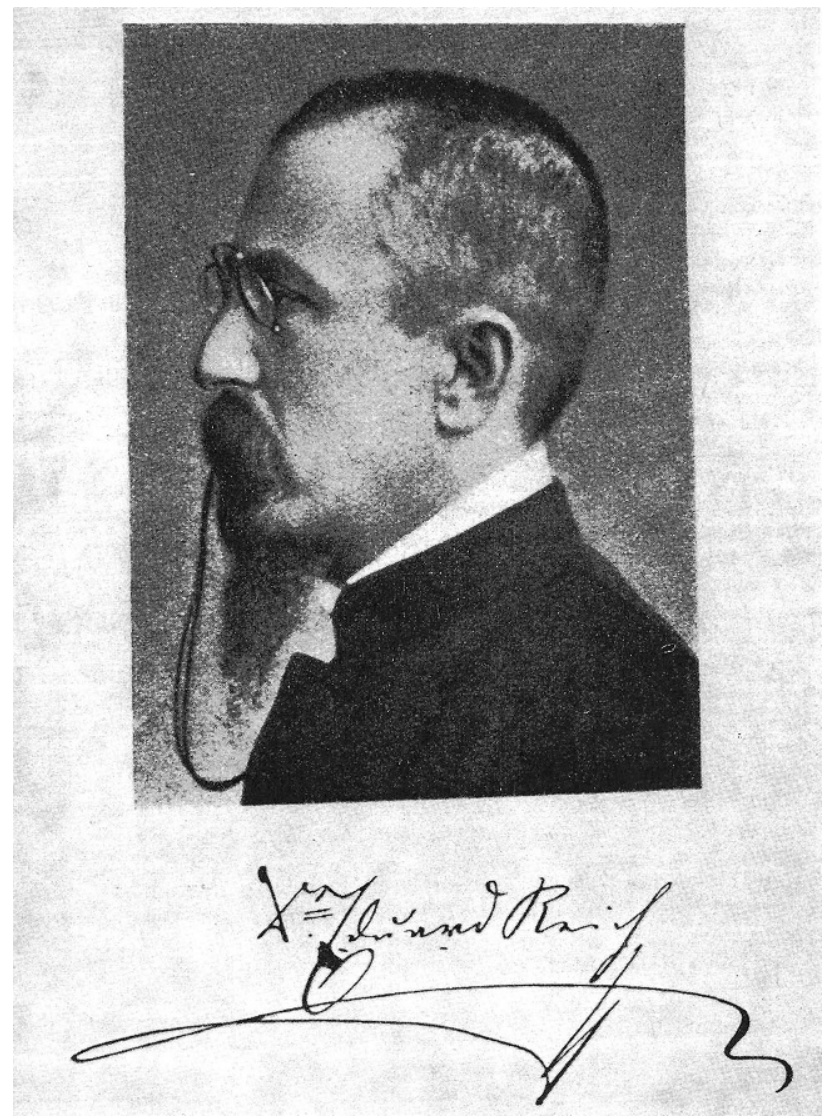

Abb. 1 Portrait aus Fischer [4]

Bei seinem Studium widmete sich Reich neben der inneren Klinik und der Medizingeschichte „ganz vorzüglich der gesamten Hygieine [sic] mit allen ihren medizinischen, naturwissenschaftlichen und politisch-moralischen Hilfs- und Neben-Disziplinen“. Unter den Hilfswissenschaften kamen ihm ganz besonders die in einem Privatinstitut besuchte Vorträge über chemische und mechanische Technologie zustatten. Seine Rigorosen absolvierte er in den Jahren 1856 und 1857. Ganz bescheiden vermerkte Reich, er selbst habe es sich niemals zugetraut, was seine Prüfer über ihn sagten, nämlich, „dass seit mehr als zwanzig Jahren kein Candidat vorgekommen sei, der mit so ausgezeichnetem Erfolge seine Examina bestanden habe".

Im letzten Studiensemester Reichs hatten sich seine bis dahin sorgenfreien Verhältnisse zum Schlechten geändert. „Bei den anstrengendsten und aufreibendsten Geistes-Arbeiten“ als Assistent am Physiologischchemischen Universitätsinstitut in ${ }^{* * * *}$ [Jena] musste er „Hunger und Kälte leiden“. Die unter diesen bösen Verhältnisse erlittenen Beeinträchtigungen hätten „den Keim zu einem Psoas-Abszess“ gelegt, an dessen Folgen Reich noch jahrelang leiden sollte.

$\mathrm{Zu}$ den äußeren Traumen kam noch, dass für die Bezahlung der Promotionstaxen von fast 150 Talern ihm und seinen Eltern das Geld fehlte und sehr reiche Verwandte „in der schmählichsten Weise“ ihre Hilfe verweigerten. In der größten finanziellen Not kam jedoch Hilfe durch den Buchhändler Ferdinand Enke in Erlangen, der das als Dissertation vorgesehene Manuskript der „Medizinischen Chemie“ um 300 Taler übernahm. So konnte Eduard Reich 1857 zum Doktor der Medizin und Chirurgie promoviert werden. Im folgenden Jahr erschien sein zweibändiges Lehrbuch „Medicinische Chemie mit Berücksichtigung der österreichischen und preussischen Pharmakopoe" [9], das er „Seinem hochverehrten Lehrer, Herrn Hofrat Prof. Dr. C. G. Lehmann, als geringfügiges Zeichen dankbarer Verehrung und aufrichtiger Hochachtung“ widmete. Die „Medizinische Chemie“ wurde übrigens ein internationaler Erfolg. So begann Reich seine „hygieinischen, ätiologischen und anthropologischen Schriften herauszugeben“ und in den folgenden Jahren an einigen Universitäten „ernsthafte staatswissenschaftliche, philosophische und historische Studien“ zu betreiben. „Immer und überall als konsultierender Arzt und Gewissensrat tätig,“ suchte er „den Zusammenhang der Physik mit der Moral zu erforschen und die Angelpunkte einer allgemeinen Anthropologie und Hygieine zu gewinnen“ [5].

Die Beziehungen Reichs zu seinen sozialen Kontakten in Jena wurden zunehmend schlechter. Er wurde, wie er meinte, „von verleumderischen Schuften in der schändlichsten Weise“ angegriffen. Und „Professor *******“ [Carl Gotthelf Lehmann, 1812-1863], war - „so große Verdienste auch um die Wissenschaft er sich erworben hatte“ - „doch so charakter-schwach, dass er den Lügen und Verleumdungen endlich im vollsten Umfang Beachtung schenkte“. Als „Ursache der niederträchtigen Behandlung“ schien Reich, dass er in seiner „Unschuld die Eitelkeit eines großen Esels verletzt" habe.

Im Herbst 1857 verließ Reich Jena, „den Ort seiner Qual“, seiner „wahren Höllen-Pein“. Selbst unter glänzendsten Verhältnissen hätte er niemals „in einem so kleinen Neste, welches einem so lächerlich kleinen Klein-Staate [Sachsen-Weimar] angehört, sich akklimatisieren können“.

Nach einer größeren Ferienreise ließ sich Reich in der kurhessischen Universitätsstadt Marburg für zwei Jahre nieder. In diesem durch „Engherzigkeit, Rohheit und Kleinlichkeit, sowie die Frömmelei“ Reichs Entsetzen erregenden „prachtvoll gelegenen, aber halb tatarischen“ Neste lernte er aber auch „sehr gute Leute“ kennen, die seinem Verweilen einen Halt gaben.

Im ersten Marburger Jahr widmete sich Reich der Abfassung seines „Lehrbuchs der Allgemeinen Ätiologie und Hygieine“ [10]. Darin stellte er sich die Aufgabe, „die ätiologisch-hygieinische Wissenschaft zu lehren, eine Disziplin, welche als solche bisher noch nicht existierte“, was wohl stimmte. Seine Überlegungen werden weiter unten mit späteren Ergänzungen dargestellt.

Das „Lehrbuch der Allgemeinen Ätiologie und Hygieine“" wurde einerseits „in den Himmel erhoben“, andererseits „in die tiefste Hölle verdammt“. Nach 
seiner eigenen Einschätzung gaben sein jugendliches Alter und sein „Bestreben, der Hygieine, dieser wahren Grundlage aller heilenden Kunst und aller menschlichen Wohlfahrt in den Ländern der deutschen Zunge Eingang und Geltung zu verschaffen“ und sie „mit der Lehre von den Ursachen der Krankheit organisch zu verschmelzen“ dem Buche ein „eigentümliches Gepräge“. Noch nach Jahren bezeichnete Reich in seiner Autobiographie [6] als Ursache der seinerzeitigen „Schmähungen“ seines Werks und insbesondere von dessen „Verurteilung und Vernichtung in Bayern“ einige von ihm als damaliger ,jugendlicher BrauseKopf“ geschriebene „eher unwesentliche und nebensächliche Ausfälle, welche gegen Pfaffen, Missbräuche etc. gerichtet sind“. Er habe es so hingeschrieben wie seine Überzeugung und sein Herz es ihm befohlen hatten. Aber „allerhand Pfaffen, bezahlte Rezensionen-Schreiber, fromme Ärzte und große Esel versuchten es, Buch und Verfasser in den Staub zu ziehen“. Jedoch die Richter seines Werks, die „nicht von Leidenschaft und Vorurteil durchdrungen waren, welche nur das Wesentliche in das Auge fassten und schäbige Privat-Interessen aus dem Spiele ließen,“ also „reinen Herzens waren“, haben mit Würde und nur mit dem Verstand geurteilt. Davon zeugen die Beurteilungen in vielen Ländern. Also wieder ein Rundumschlag gegen die vermeintlich Bösen dieser Welt.

Reich war überzeugt, dass ihm in Marburg die Widmung des Buchs an Prof. Dr. Carl Vogt (1817-1895) den größten Schaden, ja sogar die Verhinderung einer erwarteten Heirat zugefügt habe. Vogt, ein eifriger Vorkämpfer des Materialismus und Anhänger des Darwinismus, hatte sich aktiv in der Revolution von 1848 betätigt. Er war Oberst der Gießener Bürgergarde, wurde ins Frankfurter Vorparlament, in die Deutsche Nationalversammlung und ins Rumpfparlament in Stuttgart gewählt, wo er 12 Tage Reichsregent war. Schließlich musste Vogt als einer der letzten Kämpfer für die Volksgrundrechte nach Bern fliehen [11, 12].

Im zweiten Jahr im kurhessischen Marburg begann Reich sein Werk über „Nahrungs- und Genussmittelkunde“ [13]. Diese Arbeit half ihm, die heftigen Schmerzen und prekären Verhältnisse $\mathrm{zu}$ ertragen. Als dann im Frühjahr 1859 der Krieg von Österreich gegen Piemont und Frankreich ausbrach, stand Reich „auf der Seite der Franzosen und Italiener“. Er bezichtigte Österreich, Kurhessen und die angrenzenden Länder und „mikroskopischen Staaten“ einer Dummheit, die „Rhinozerossen und Büffeln selbst Schande gemacht" hätte. Diese Haltung ließ Reich viel Unangenehmes und Verletzendes erfahren und es erschien der Aufenthalt in Marburg für ihn wie auch „für jeden politisch Gesunden geradezu unmöglich“.

1859 entschloss sich also Reich, Marburg zu verlassen und nach Göttingen zu übersiedeln. Auch dort hatte er zuerst „eine sehr schwere Zeit“ und „litt außerordentlich“. Dies besserte sich, als ihm von der Verlagshandlung Vandenhœck \& Ruprecht für sein begonnenes Buch ein Vorschuss gewährt worden ist.
Er fand eine gesunde Wohnung. Die sehr freundliche Aufnahme bei den Gelehrten und die Großherzigkeit in der Bevölkerung gaben ihm „neue Lebens-Hoffnungen“. In der großen Bibliothek kamen ihm die Oberbeamten sehr gefällig entgegen. Doch ein „sehr pfäffisch gesinnter, sehr kleinlicher, beschränkter und seiner freien Richtung sehr feindlich gegenüber stehender Unter-Beamter war die Ursache, warum die Benützung der Bibliothek zuletzt so sehr ihm verleidet wurde“.

Reich hatte schon länger die Absicht, sich der akademischen Laufbahn zuzuwenden. An deutschen Universitäten verhinderte dies bisher der Mangel an nötigen Mitteln für die Taxen wie auch „teils seine [Geistes-]Richtung, teils seine Nationalität, teils endlich hier und da die Verletzung der Eitelkeit verschiedener alter und junger Esel“ (Abb. 1).

So verließ Reich 1860 Göttingen und kam auf einem großen Umweg mit vielen Empfehlungen von lieben Göttinger Bekannten nach Bern um sich zu habilitieren. In seinem Gesuch an die Hohe Erziehungs-Direktion in Bern vom 20. April 1860 drückt er seine Überzeugung aus, „dass die gesamte Hygieine nicht nur für den Arzt und Sanitäts-Beamten von der äußersten Notwendigkeit ist, sondern auch dem Erzieher, Lehrer, Richter u.s.w. tausende von Anhaltepunkten für das Leben und Wirken bietet, dass daher deren Lesung auf Akademien von sehr großer Bedeutung ist“. In der nächsten Fakultätssitzung berichtet der Dekan, dass Dr. Reich in seinen Publikationen „bei jeder Gelegenheit über die Geistlichen in etwas roher Weise losziehe und sich selbst dadurch dekreditiert habe“. Die medizinische Fakultät kam zum Schluss, dass der Aspirant offenbar „der betreffenden Fächer des medizinischen Wissens vollkommen mächtig sein muss“, jedoch für die Beurteilung seiner Lehrfähigkeit eine Probevorlesung zu halten hat. In dieser sprach Reich am 12. Mai 1860 über den „Kaffee in hygienischer Beziehung“. In der Schlussansicht der Fakultät vom 18. Mai wird zwar angegeben, dass der Probevortrag „in gewissen Beziehungen Einiges zu wünschen übrig ließ“, damit aber die Abweisung des eingereichten Gesuchs nicht motiviert werden könne. Hierauf erteilte der Direktor der Erziehung des Kantons Bern Reich am 21. Mai die Venia docendi für die gesamte Hygieine [14].

Als Dozent (Professeur agrégé) erging es Reich wie seinen „die Hygieine französisch lesenden Kollegen und vielen anderen braven Männern, die vor ihnen die Hygieine gelehrt“ hatten, dass sie nämlich fast gar keine Zuhörer bekamen. Die Studenten hätten kaum Interesse selbst für Fächer, die geprüft wurden. Auch die Professoren, insbesondere diejenigen aus Deutschland, beurteilte Reich sehr negativ. Neben seinem Fach hielt Reich auch Vorlesungen über Geschichte und Enzyklopädie der Medizin und über Toxikologie.

Nach der Habilitation zog Reich in eine bessere Wohnung im Haus einer ehemaligen Patrizierfamilie. Das rasch gediehene Liebesglück mit der Filia hospi- 
Abb. 2 Manifest an die deutsche Nation!

\section{Mtanifeft an Die Dentide Mtation!}

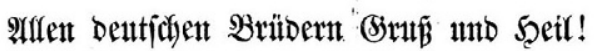

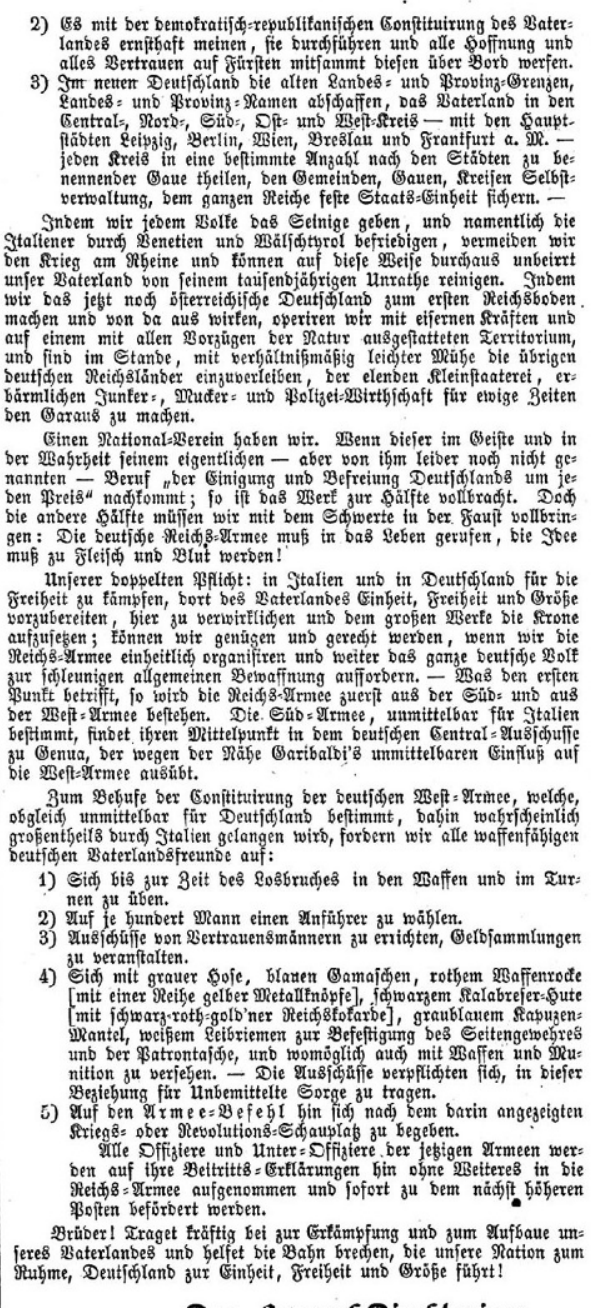

Das generaf=Direfitorium. talis wurde durch eine „alte Großmutter des Teufels“ erfolgreich hintertrieben. Deswegen wechselte Reich im Herbst 1860 seine Unterkunft. Dies verschaffte ihm vielleicht die notwendige Zeit und Ruhe für gesteigerte politische Aktivität. So hielt er im Deutschen Bildungsverein Vorträge über Gesundheitspflege, aber auch über Geschichte und Politik.

\section{Reich als verhinderter Revolutionär}

Von seiner großen Sympathie für Deutschland veranlasst, wollte Reich am liebsten die Vereinigung seines Vaterlandes Mähren mit dem Deutschen Reich, eine Meinung, die er allerdings schon bald [1864] als „unheilvolle Täuschung“ bezeichnete. Doch vorerst suchte er in der Schweiz für ein von ihm verfasstes Rundschreiben mühsam einen Drucker. Im April 1861 konnte er endlich in Solothurn sein „Manifest an die deutsche Nation“ [15]! drucken lassen. Ein Exemplar dieses einseitigen, jedoch dichten „Pamphlets“, wie es Reich drei Jahre später [6] bezeichnete, wurde jetzt [2015] durch intensive Bemühungen von Frau Mag. Wilma Buchinger der Österreichischen Nationalbibliothek im Staatsarchiv des Kantons Bern gefunden.

Nach der Anrede „Allen deutschen Brüdern Gruß und Heil!" wird über die Sonne der Freiheit geschwärmt, die über dem Lande der italienischen Leidensbrüder aufgegangen sei. Dessen heldenmütige Söhne hätten „endlich das Joch zerbrochen und den Anfang zur Befreiung Europas aus den Klau- 
en der Tyrannen und Pfaffen gemacht“. „Indem wir mit ihnen, mit den Ungarn, Polen und Südslaven vereinigt, das vom Weltgerichte tausendfach verurteilte Haus Habsburg-Lothringen vom Throne werfen und unschädlich machen" werden die Grundfesten des einheitlichen und freien Deutschland geschaffen, dessen geplante Struktur beschrieben wird. In dieser Tonart geht das Schriftstück weiter. Aus der Pflicht, in Italien und Deutschland für die Freiheit zu kämpfen, erwüchse die Notwendigkeit der „Konstituierung der deutschen West-Armee“. Zu deren Aufstellung werden „alle waffenfähigen deutschen Vaterlandsfreunde“ aufgefordert. Es folgen darauf genaue Angaben, die bis zur Beschreibung der Knöpfe des Waffenrockes gehen. Unterzeichnet ist das datumsfreie Manifest mit „Das General-Direktorium“ (Abb. 2).

Der kurze Berner Polizeibericht [16] stellte fest, dass das Manifest von einem Dr. Eduard Reich aus Olmütz verfasst worden war und dass die vermeintliche Verbindung und das Generaldirektorium bloß aus ihm allein bestehen. „Da diese kleinen Mittel gegenüber der großen Aufgabe ziemlich ungefährlich erscheinen“, beschränkte sich die Polizei auf die Androhung einer Landesverweisung im Wiederholungsfall oder bei politischen Umtrieben. Rückblickend bezeichnet Reich in seiner Autobiographie „diese ganze Manifest-Geschichte als eine große Torheit" [6].

Trotz der Abweisung des Wunsches der Österreichischen Regierung an die Eidgenossenschaft, diese möge gerichtliche Erhebungen für eine Anklage Reichs wegen Hochverrats anstellen, sah dieser sich gezwungen, die Schweiz zu verlassen. Jetzt nahm er, nach mehrmonatlichem Leiden an seinem Bein gesundheitlich „fast an den Rand des Grabes gebracht“, die Einladung des Dekans der Straßburger medizinischen Fakultät an. Ohne das nötige Kapital zog er nach Straßburg, das er aber wegen eines „Ereignisses rein privater Natur“ trotz seiner großen Vorliebe für Frankreich bereits im Sommer 1861 verließ.

In seiner nächsten Station, in Coburg, blieb er nur zwei Wochen. Bevor er jedoch nach Gotha weiterreisen konnte, musste er sich noch Geld für die Bezahlung seiner Unterkunft ausleihen.

Anfang September 1861 in Gotha angekommen erhielt Reich von Herzog Ernst II. von Coburg-Gotha die Zusicherung hindernisfreien Aufenthalts und die Erlaubnis, die herzogliche Schlossbibliothek benützen zu dürfen. Dort fand er bei den Beamten dieser öffentlichen Bibliothek eine besondere Zuvorkommenheit. Aber auch hier konnte er nicht umhin, Negatives festzustellen, in dem er bekrittelte, dass die Gelehrten sich auffallend wenig um die wertvolle Bibliothek kümmern. Die Ursache sah er im „Übermaß und Unwesen des gegenseitigen Besuchens“ und im „mit wahrhaftiger Todesverachtung betriebenen Bier-Kultus“. Reich selbst aber hielt sich an die „Grundsätze der strengsten Hygieine, denn er lebte in Armut, Keuschheit und im Dienste der Wissenschaft." Er war Vegetarier, ba- dete täglich zweimal in kaltem Wasser und wich Äußerlichkeiten stets aus [17].

Reich hielt Vorträge über „die Lehre vom Menschen im Allgemeinen und von der Gesundheit.“ „Alles, was dem Menschenwohle feindlich gegenüberstand, bekämpfte“ er so heftig, dass ihm „grimmige Feinde erwuchsen“, die ihn verderben wollten. Im privaten Umfeld machte er sich durch politische Äußerungen manche Feinde, die ihn bekämpften und seine Werke als geistlose Schreibereien und als Pestgift verschrien. Er klagte, dass er als „der politisch Gebrandmarkte“ und auch durch seine „radikale wissenschaftliche Richtung“ in der Unterbringung einiger seiner Werke gehindert, sich kein „festes Brod“ [Einkommen] verschaffen konnte. Seine Geldnot war so groß, dass einige seiner Gläubiger bei der Polizei seinen Pass beschlagnahmen ließen.

Aus seiner fürchterlichen Geldnot hätte Reich vielleicht die Tätigkeit als praktizierender Arzt helfen können, doch waren dafür der Erwerb der Gothaischen Staatsbürgerschaft und eine Examinierung durch Praktiker nötig, die nach seiner Meinung „um 30 Jahre hinter der Wissenschaft zurück sind“. Dies war für Reich aber eine Zumutung für sein Ehrgefühl, hielt er sich doch als „Gelehrter von Rang und Namen“, als „damals schon eine international anerkannte Autorität in der wissenschaftlichen Hygieine“.

Im Spätsommer 1862 begann Reich die Ausarbeitung seiner „Geschichte, Natur- und GesundheitsLehre des ehelichen Lebens" $[6,18]$. Es sollten die Grundlagen des „normalen Fortpflanzungs-Lebens in Staat und Gesellschaft“ ermittelt werden, um das „Institut der Ehe“ aus der bisherigen „ausschliesslichen General-Pacht“ der „natur-unkundigen Theologen und Rechts-Gelehrten“ in die Hände der „hygieinischen Aerzte zu legen“. Die Reaktionen auf dieses Buch waren unterschiedlich. Es wurde in Fachzeitschriften, auch in der Wiener Medicinischen Wochenschrift, gelobt, aber eine „gewisse Klasse“, insbesondere „Ultramontane und andere Nacht-Eulen (in schwarzen Kutten) spieen Gift und Galle dagegen“.

Nachdem Reich zum Bibliothekar der herzoglichen Bibliothek in Coburg ernannt worden war, ging er im Mai 1869 die Ehe mit Mathilde Loewel, der Tochter eines Finanzrates, ein, die ihm bis zu ihrem Tod 1887 neun Kinder schenkte [17]. Die Bibliothekarsstelle bekleidete er bis 1873, war jedoch meist bei halbem Gehalt beurlaubt. So konnte er verschiedene große und kleine Reisen ins Ausland, in Deutschland und auch in seine Heimat Mähren unternehmen, wo sein politisches Abenteuer den für ihn unerwarteten Ausgang nahm. Nach der „Press-Amnestie“ vom 31. Juli 1865 meinte er sich beim Besuch seiner Eltern in Olmütz im Feber 1866 vor Verfolgung sicher. Er wurde jedoch durch einen Irrtum der k. k. Behörden elf Tage lang wegen Hochverrats inhaftiert, ein Vorkommnis, das auch in der Wiener „Presse“ mehrmals kommentiert wurde [19]. 
Noch als herzoglicher Bibliothekar angestellt, übersiedelte Reich mit Familie nach Kiel, wo er ,in den gelehrten Kreisen“ sehr freundlich aufgenommen wurde. Auf sein formelles Gesuch an die medizinische Fakultät um die „Venia legendi für die gesamte Hygieine" wurde in Gotha nachgefragt. Nach Eintreffen einer „empörenden Verläumdung“ wurde ein negatives Gutachten über ihn durch „die (mit einem die Gesundheitspolizei der Abzugscanäle und Schlachthäuser tradierenden Privatdocenten und Kreisphysiker innig befreundete) Fakultät“ erstellt (ein mildes Beispiel für seine Ausdruckweise; [5]). „In sehr wenig elastischen Ausdrücken" forderte Reich seine Eingaben zurück.

In der ersten Hälfte von 1870 ließ sich Reich „durch falsche Vorspiegelungen eines in Würzburg wohnenden von ihm massgebend gehaltenen“ Mann verführen, mit Frau und neugeborenem Sohn Napoleon nach Würzburg zu übersiedeln. Dort war dieser Ratgeber jedoch nicht aufzufinden, sodass sich die Familie nach wenigen Tagen über Coburg nach Erlangen begab. Reich stellte dort fest, dass er „in seinem ganzen Leben und in der ganzen Welt keine so grossen und steifen Schwachköpfe“ gesehen hat. Er beendete in Erlangen noch sein zweibändiges Werk „System der Hygieine“ und übersiedelte danach auf das zu Bayern gehörende Schloss Banz bei Coburg. Im Herbst 1871 wollte Reich direkt nach Coburg übersiedeln, musste aber wegen einer Intrige nach Weimar ziehen. Mangels einer geeigneten Wohnung in der Stadt verbrachte die Familie den Winter in der HerrnhuterGemeinde Dietendorf. Im Sommer 1872 hauste die Familie in Sonderhausen, von wo sie im Herbst nach Rostock übersiedelte. Dort verloren sie „irdisches Gut“ durch einen der dortigen zahlreichen Betrüger. Reich fühlte sich trotzdem in Rostock wohl. Er kündigte im folgenden Jahr seine Anstellung als herzoglicher Bibliothekar.

Das folgende persönliche Schicksal Reichs lässt sich Mangels weiterer Autobiographien nur ganz oberflächlich nachzeichnen, da das „Reich-Archiv“ des Deutschen Hygiene-Museums mit allen Unterlagen durch einen Luftangriff auf Dresden im 2. Weltkrieg vernichtet worden ist.

Nach dem Tod seiner Frau Mathilde im Jahre 1887 heirate Reich 1892 Helene Stavenow, die Tochter eines Hamburger Kaufmannes [17]. Als weitere Aufenthaltsorte sind 1891 Blankenese, 1892 Biebrich am Rhein, 1893 Scheveningen (Niederlande) und 1902-1914 in Belgien die Städte Ostende (1902), Nieuport-Bains (1903) und La Panne (1909) bekannt. Anfang September 1914, als Ausländer zum Verlassen Belgiens gezwungen, flüchtete er unter Zurücklassung seines ganzen Besitzes, einschließlich seiner großen Bibliothek, in die Niederlande, wo er am 1. Februar 1919 in Muiderberg bei Amsterdam starb [11, 17]. Reich ist zwar in den üblichen medizinischen biographischen Referenzwerken mit langen Listen seiner zahlreichen Bücher und Fachartikel enthalten, man findet aber außer seinen autobiographischen Angaben [5, 6] nur zwei kurze biographische Bearbeitungen $[8,20]$ in der medizinischen Literatur.

Die in Bern begonnene akademische Laufbahn fortzusetzen hatte Reich dreimal vergeblich versucht. So richtete er am 18. November 1867 an den Münchner Hygieniker Max von Pettenkofer (1818-1901) die Bitte, ihm zu einer Professur zu helfen. Er betonte seine Kenntnisse nicht nur der Chemie und Physiologie, sondern auch der unerlässlichen „politischmoralischen Wissenschaften und der Anthropologie“ [21]. Am 3. August 1871 wandte sich Reich an den Zoologen und Naturphilosophen Ernst Haeckel (1834-1919), wobei er auf seine Verhältnisse „eines armen Gelehrten“ hinwies, der „durch CollegienGelder den Betrag der Miethe u.s.w.“ decken muss. Da die medizinischen Fakultäten Deutschlands nicht zu Vorlesungen in seinem Sinne über Hygieine, über physiologische, philosophische und soziale Anthropologie bereit seien und diese Disziplinen „viel mehr in die philosophische, denn in die medicinische Fakultät passen“, wendete er sich an ihn [22]. Der Versuch der Wiederaufnahme einer akademischen Lehrtätigkeit zuletzt in Kiel war ebenfalls erfolglos geblieben.

\section{Reichs Prinzipien der Hygiene}

Reich hat die Prinzipien seiner Auffassung der Hygiene 1862 im Buch „Volks-Gesundheits-Pflege“ [23] und 1870-1873 umfänglicher im zweibändigen Lehrbuch „System der Hygieine“ [24] und im „Grundriss der Hygieine zum Gebrauche für akademische Vorlesungen und zum Selbstudium“ [25] ausführlich dargelegt. Ergänzt wurden diese Werke durch eine äußerst große Anzahl von weiteren Büchern und Artikeln, die Ergänzungen und Modifikationen, aber auch sehr viele Wiederholungen enthalten. Diese Literatur habe ich, soweit es mir erforderlich erschien, in die Besprechung von Reichs Grundstruktur der „Hygieine“ eingebaut und mit ihren Literaturangaben versehen. In der Folge habe ich jedoch den heute üblichen Begriff „Hygiene" und in Zitaten die derzeitige Rechtschreibung verwendet.

Reich sah prinzipiell als Aufgabe der Hygiene nicht nur einer Erhöhung der Arbeitskraft, sondern die Hygiene will die Menschen „vergeistigen, veredeln, verschönern und gesund machen“. „Weisheit und Tugend, Glückseligkeit und Gesundheit Aller, dies ist das Endziel der Hygiene." Die Hygiene hat dabei mit dem ganzen Menschen sowie mit dessen einzelnen Organen und Systemen zu tun, es muss also wer „die Hygiene richtig begreifen will“, die Anthropologie, die Physiologie und die Psychologie richtig verstehen. Da die Hygiene aber nicht nur die Gesundheit zu erhalten lehrt, sondern auch Krankheiten zu verhüten, ist die Kenntnis der Ätiologie der Krankheiten unumgänglich. Und „so wie die Hygiene des Individuums auf die Naturlehre des Individuums sich gründet, so gründet die Hygiene der Gesellschaft sich auf die Naturlehre 
der Gesellschaft“, also auf Nationalökonomie, Bevölkerungslehre, Politik, Polizeiwissenschaft und Statistik.

Nach Reichs Auffassung [10, 26] sind die Lebensprozesse die Folgen der Einwirkung der Außenwelt auf den Organismus. Wenn die „biogenetischen Faktoren“ [Organismus, Außenwelt] derart zusammenwirken, dass der Stoffwechsel ohne Störung und mit „allgemeinem Wohlsein, Gefühl von Kraft und Lust“ abläuft, sprechen wir von $\mathrm{G}$ e s u n d h e i t. Das bedeutet die Erhaltung des „vegetativ-irritablen, sensiblen, wie geistig-sittlichen Wohls des Einzelnen wie ganzer Nationen“, das Bewahren vor gemeinen Affekten und Leidenschaften und vor allen Einflüssen, die das somatische und psychisch-moralische Ich vernichten. „Je mehr Nerven- und Seelenkraft ein Mensch besitzt, desto weniger leicht werden ihm Krankheiten gefährlich“ und es stellt sich „ein ganz bestimmtes Verhältnis zu der Erblichkeit von Eigenschaften des Körpers und des Geistes, zu den ererbten Anlagen für Leiden und Gebrechen“ ein [27].

„Um gesund zu sein, müssen wir gut und weise sein: um gut und weise zu sein, müssen wir gesund sein. Weise werden wir durch Unterricht, gut durch Erziehung, gesund durch Pflege." Erziehung und Unterricht sind Gesundheitspflege der moralischen Qualitäten, Pflege schlechthin ist die Gesundheitspflege der physischen Qualitäten, also des materiellen Daseins. „Weil der Mensch die Einheit des Physischen und Moralischen ist und weil das Moralische nur eine besondere Erscheinungsweise des Physischen ist: darum sind Erziehung, Unterricht und Pflege Eines, sie sind Gesundheitspflege oder Hygiene."

In ihrer Art und Intensität abweichende Wechselwirkungen zwischen Organismus und Außenwelt bezeichnet Reich als $\mathrm{K} \mathrm{r}$ a $\mathrm{n} \mathrm{k} \mathrm{h} \mathrm{e} \mathrm{i} \mathrm{t.} \mathrm{Ihre} \mathrm{Ursache} \mathrm{kann}$ im Organismus selbst bestehen durch „Herabsetzung der Kräfte des organischen Haushalts, des Nervensystems und der Seele“. Sie kann aber auch in der Art und Stärke von physischen oder moralischen Umwelteinwirkungen liegen. Aber auch an sich normale Außeneinwirkungen können bei Veränderungen im $\mathrm{Zu}$ stand des Menschen pathogen wirken. Zwischen Gesundheit und Krankheit bestünde nur eine „graduelle Verschiedenheit“, aber kein Gegensatz, da physiologische und pathologische Prozesse nach denselben Gesetzen, aber nur unter verschiedenen Bedingungen abliefen.

Zum Verständnis für das „physiologische Leben“ der Einzelwesen wie auch ganzer Nationen mit den verschiedenen Zuständen und Bedürfnissen sowie für die Erkenntnis von deren Verhältnissen zur Außenwelt [heute: Umwelt] bedürfe es nach Reichs Überzeugung nicht nur der Kenntnisse der Physiologie, sondern auch der Naturgeschichte, Physik, Meteorologie, Geologie, Chemie, Geographie, Geschichte und Statistik. Alle diese Probleme und Umstände der Entstehung von Krankheiten beschreibt die Ä t i o l o g i e.
Die H y g i e n e oder G e s u n d h e i t s p f l e g e ist „die Philosophie, die Wissenschaft und die Kunst des normalen Lebens; sie lehrt Krankheiten zu verhüten, das gesamte physische und moralische, das persönliche und gesellschaftliche Wohl zu erhalten. Reichs Vision vom Ergebnis des „mächtigen Ineinandergreifens“ von Ätiologie und Hygiene ist die „Erhaltung des Wohls des Einzelwesens wie ganzer Völkerschaften“, das Bewahren „vor gemeinen Affekten und Leidenschaften und vor all den Einflüssen, die das somatische und psychisch-moralische Ich vernichten“; die „innige Wechselwirkung beider Doktrinen“ „mildert Pandemien und sporadische Krankheiten, erhält die Gesundheit, verlängert das Leben und trägt so zur Entstehung gesunder Nachkommen bei“. Gestützt auf die Kenntnis der Krankheitsursachen und der Natur des Menschen, der sozialen Verhältnisse und der sittlichen Bedürfnisse, sucht die Hygiene überall das Gute zu fördern und das Böse zu verhindern, leitet zur Tugend ebenso wie zur Glückseligkeit.“ So sollen „Krankheiten unmöglich, die Medizin überflüssig“ gemacht werden. Die Hygiene hat als Endziel aber nicht bloß die Erhöhung der Leistungsfähigkeit der Bevölkerung für irgendwelche ökonomische oder militärische Zwecke, sondern sie will die Menschen „vergeistigen, veredeln, verschönern, gesund machen“ für die Erlangung von „Tugend, Glückseligkeit und Gesundheit Aller“.

Reich beschreibt mit seiner ideellen Vorstellung bereits 88 Jahre vor der Definition der Gesundheit durch die Weltgesundheitsorganisation [28] als einen „Zustand völligen körperlichen, seelischen und sozialen Wohlbefindens und nicht nur die Abwesenheit von Krankheit und Schwäche“.

Der Hygieniker müsse zur Erfüllung aller dieser Aufgaben die Ätiologie der individuellen und sozialen Krankheiten erkennen. Das heißt für Reich, der Hygieniker muss nicht nur die Naturlehre des Individuums, also Physiologie, Psychologie und Anthropologie, sondern auch Naturgeschichte, Physik, Meteorologie, Geologie, Chemie, Geographie, Geschichte und Statistik, richtig verstehen und auch die Naturlehre der Gesellschaft, also Nationalökonomie, Bevölkerungslehre, Statistik, Politik und Polizeiwissenschaft, kennen.

Nach Reichs Vorstellung muss die „ätiologisch-hygienische Wissenschaft“ zum Gemeingut aller Menschen gemacht werden. Als geeignete Mittel hierfür erschienen ihm außer dem Unterricht auch Predigten über Ätiologie und christliche Moral sowie Gesetze und „staatliche heilbringende Einrichtungen“. Dabei dürfen die Politiker nicht in den Prozess der „Menschen-Bildung“ störend eingreifen, sondern müssen ihn fördern. Aus der so größeren Menge Studierender wäre es ein Leichtes, viele „freidenkende Individuen aus der Menge herauszufinden, denn es komme darauf an, Wissenschaft und Staat in die Hände denkender Menschen zu legen“. Beschränkte Köpfe müssten jedoch „ihren Unterricht beständig in Sonn- 
tagsschulen und ähnlichen Anstalten genießen und es müssen diese Institute unter der Leitung einer Medizinal-Zentralbehörde, unter der speziellen und persönlichen Leitung eines tüchtigen Arztes stehen“. Die so erreichbare „hygienische Erziehung des ganzen Menschen" ist die Voraussetzung des Nutzens der Heilkunst. Allerdings beklagt Reich auch den Mangel an Kenntnissen der Ätiologie und Hygiene bei den Ärzten, was er auf das Versagen der Universitäten zurückführt. Vielfach werden einschlägige Vorlesungen gar nicht angeboten und wo dies doch der Fall ist, werden diese wegen Mangels an Hörern nicht abgehalten. Dagegen würde eine landesfürstliche Verordnung der zwangsweisen Belegung und Prüfung dieser Fächer Abhilfe schaffen. Darüber hinaus wäre es wünschenswert, dass sich jeder Bewerber um eine Staats- oder geistliche Anstellung, sowie jeder Heildiener und jede Hebamme einer Prüfung aus den wichtigsten Lehren der populären Physiologie, Ätiologie und Gesundheitspflege unterziehen müsste.

In Vorwegnahme der zukünftigen KrankenhausHygiene erkannte Reich, dass auch die Therapie überall der Hygiene bedarf, denn „was nützen alle Operationen ohne Ventilation, Desinfektion u.s.w. der Kranken-Räume?“ „Wo die Medizin wirksam sein soll, kann sie dies nur in der Voraussetzung, dass die Hygiene tätig ist.“ Die Hygiene verlangt also vom therapeutisch arbeitenden Arzt, über das Heilen von Krankheiten hinausgehend, das Verhüten der Leiden ernst zu nehmen.

Reich unterteilt die Hygiene entsprechend ihren Aufgaben in eine Moralische, Soziale, Diätetische und Polizeiliche Hygiene. Weiters erwähnt er noch die von Ribes [29] angegebene Therapeutische Hygiene, welche die Anwendung der Gesundheitspflege zum Heilen von Krankheiten darstellt.

\section{Die Moralische Hygiene}

Die Moralische Hygiene hat die Aufgabe, für den Einzelnen und für die Gemeinschaft die moralische Gesundheit zu erreichen und auf Dauer zu erhalten. Sie muss dazu das geistige und sittliche Leben, also die durch das Gehirn des Menschen gesteuerten Vorgänge des Geistes, der Handlungen, des Gemüts und der Leidenschaften so regulieren, dass sich für den Einzelnen und die Gemeinschaft der normale Zustand ergibt. Für diese Aufgaben bedient sich die Moralische Hygiene der Erziehung, des Unterrichts und der Religion.

„Die moralischen Handlungen erfolgen nach unwandelbaren Naturgesetzen“. Sie sind Ausdruck des Triebes der Selbsterhaltung und der Fortpflanzung. Ihnen gegenüber hat „der sogenannte freie Wille keine Bedeutung; er vermag nur die Form der Vollziehung, nicht den Akt selbst zu beeinflussen“.

Das moralische Leben ist durch Elend und Armut, aber auch durch Ungerechtigkeiten und manche Traditionen bedroht. Das Elend zerstört die physischen und moralischen Kräfte, es hemmt insbesondere die Entwicklung von Wohlverhalten, Nächstenliebe und Gemeinsinn.

Zur Beeinflussung der moralischen Handlungen darf nicht nur an den Willen der Menschen appelliert werden, sondern es müssen auch deren natürliche Bedürfnisse befriedigt werden. Dazu gehören Verbesserungen der gesellschaftlichen Einrichtungen, der Sitten und Gebräuche und die Aufklärung [Erziehung, Unterricht, Berufsausbildung] zu Gunsten des Einzelnen und der Gesamtheit.

\section{Die Leidenschaften}

Unter den einzelnen Aspekten der Moralischen Hygiene stellt Reich die Leidenschaften an prominente Stelle. Diese beschreibt er als intensive mit dem Ich in Widerspruch stehende Vorstellungen, die mehr oder minder starke Spannungen erzeugen, welche den Wunsch nach Ausgleich auslösen. Die normalen, gesunden Leidenschaften harmonieren mit der Gesundheit der Seele, die bösen, krankhaften vernichten diese. „Bei allen Leidenschaften beherrscht ein gewisses Etwas die Kräfte der Seele und wirkt auf die rein körperlichen Funktionen hinüber“. Zuletzt wirkt die Leidenschaft entkräftend, verdirbt das Temperament und degeneriert den Charakter. Damit können die Leidenschaften „zu Zerstörern der Gesundheit des Einzelnen und der bürgerlichen Gesamtheit, zu Veranlassungen blutiger Taten im privaten Leben und in der Weltgeschichte" werden [2].

Reich stellt lange, durch reichliche Literaturzitate unterstützte Betrachtungen an über die Ausprägungen der Leidenschaften bei den einzelnen Temperamenten, bei Mann und Frau verschiedener Altersstufen und Gewohnheiten, auch in Abhängigkeit von Stand, Beschäftigung, Ehelosigkeit, Diät [Gesundheitszustand], Klima, Religion, Vererbung und Politik. Er schildert als Leidenschaften Liebe, Selbstsucht, Hass, Zorn, Neid, Langeweile, Spiel, Trunk- und Fresssucht, Heimweh und übermäßiger Trieb zu Geistestätigkeit und noch weitere Gefühlsregungen.

Zur Regulierung guter und zur Verhütung böser Leidenschaften müssen alle Mittel der körperlichen Hygiene, der intellektuellen und moralischen Erziehung, der Religion und der Politik auf eine Stärkung des Willens hinwirken [2]. Eine wichtige Voraussetzung ist die Sicherheit des Lebensunterhalts. Dazu sind auch gute politische Verhältnisse nötig, denn ,je mehr die Parteien auf gegenseitige Verfolgung, Prinzipienreiterei, Phraseologie, Rechthaberei u.s.w. bedacht sind, desto ungünstiger werden die moralischen Verhältnisse beeinflusst, desto schlimmer die Leidenschaften aufgeregt“.

Die allgemeine Zufriedenheit der Menschen mit ihrer Lebenslage ist die wesentliche Grundlage des moralischen Wohlseins und damit der Regelung der Leidenschaften und somit auch einer Hygiene des Geistes und des Herzens. 


\section{Das geistige Leben}

Im zweiten Abschnitt der Moralischen Hygiene behandelt Reich das geistige Leben. Er meint damit die „Gesundheitspflege des Gehirns“, das heißt die „der Natur gemäße Kultivierung des Geistes“. Gemeinsam mit dieser „Erziehung im weitesten Sinne des Wortes" muss immer die Pflege der Gesundheit gehen, sonst „weicht das geistige Leben bald von der Norm ab und gestaltet sich mehr oder weniger krankhaft". Seine Intensität und Extensität hängen von der jeweiligen Individualität ab, also insbesondere von Alter, Geschlecht, Konstitution und Temperament. Im Laufe des Lebens erweitert sich der Kreis der Ideen, der Verstand entwickelt sich und überwiegt in einem gewissen Alter die Phantasie. Wegen der allfälligen Schädlichkeit der Geistesarbeit solle ein Jeder nur so viel Kopfarbeit verrichten, wie er ohne Schaden leisten kann. „Auch die frühzeitige Anstrengung des Geistes [im Kindesalter] hat einen sehr nachteiligen Einfluss auf die physischen und moralischen Kräfte“.

Elend und Verwahrlosung zerstören die Geistestätigkeit und machen den Menschen zum Idioten. Gute soziale Verhältnisse dagegen sind die Voraussetzung für reges geistiges Leben, da man weniger an die Befriedigung der Lebensnotwendigkeiten denken muss. Einfluss auf das geistige Leben haben aber auch „Naturerscheinungen“ wie Gebirge, Gewässer, Gewitter und Klima, also die Umwelt. Von großer Bedeutung sind auch Arbeit und Beschäftigung, die „dem Arbeiter die Mittel in die Hand geben, sich und seine Nachkommen vor der Versumpfung zu bewahren“.

Als oberste Aufgabe der Hygiene des geistigen Lebens bezeichnet Reich die Verhinderung von Geistesstörungen, wobei er die damaligen Auffassungen der Psychiatrie diskutiert.

\section{Die Erziehung}

Als dritten Grundpfeiler der Moralischen Hygiene sieht Reich die Erziehung, von der mehr als von anderen äußeren Einflüssen Gesundheit und Wohlfahrt abhängen. Der „kerngesunde, lebenskräftige Mensch, der halbwegs gut geleitet und nicht durch Erziehung, Schule u.s.w. verdorben wurde“ [27], hat keine Neigungen zu Gewalttätigkeit, Rachsucht, Lastern und Verbrechen. Ohne Erziehung können sich aber diese bösen Leidenschaften und Triebe entwickeln.

„Aber ein Übermaß unfruchtbaren Wissens und Aufspeicherung von Tatsachen ohne Kraft geistiger Verwertung und praktischer Anwendung" wirken schädlich durch Verkleinerung des Spielraums des Denkens und Lähmung des Handelns. „Völker wie Einzelne, denen die Schule ein Übermaß von Kenntnissen einprägte, sind schwach im Denken, linkisch und unbeholfen im Handeln“, sie sind gleichgültig für das Gemeinschaftsleben und hängen an unpraktischen Normen.
Die Kinder sind mit Bestimmtheit und Strenge unter humanem Entgegenkommen und liebevoller Behandlung zu erziehen und dürfen nicht durch Züchtigung und andere physische Handlungen genötigt werden. Zur Erreichung der moralischen Gesundheit ist der Wille der Kinder zu erziehen, wo er fehlt ist er $\mathrm{zu}$ wecken und wo er allzu stürmisch braust ist er $\mathrm{zu}$ dämpfen. Von der größten Bedeutung für den guten Erfolg des Unterrichts bleibt immer seine Freiheit. Je mehr die Schule von dazu nicht Befähigten beeinflusst wird, desto weniger ist der Lehrer im Stande, zu individualisieren und im Geiste der Wahrheit und einer echten Praxis zu unterrichten. „Die Freiheit der Lehre ist die beste Bürgschaft des Gedeihens wahrer Gesittung."

Ganz wesentlich ist es, den Eltern das Interesse für die Bildung der Kinder beizubringen und ihnen auch die Mittel dafür bereitzustellen. „Die geringste Steuererleichterung vermehrt die Zahl der Schreib- und Lese-Kundigen im Staate stärker als eine Legion von Schulmeistern.“

\section{Religion und Sittlichkeit}

Die Besprechung von Religion und Sittlichkeit beschließt das Kapitel der Moralischen Hygiene. Im ausführlichen Text kann Reich seine auch sonst oft geäußerten Angriffe auf Geistliche und Religionsausübungen unterbringen, die hier nicht weiter ausgeführt werden.

Er anerkennt aber den Zweck der Religion, das gesellschaftliche Gleichgewicht zu erhalten und dem Einzelnen in den Wechselfällen des Lebens zur Stütze zu dienen. Je nach der Größe der Geistesbildung und moralischen Kraft, ist sie dem Einen mehr Moral, für den anderen mehr Glauben, für den Philosophen nur Moral, für den „Pfaffenknecht“ nur Glauben; demnach für einen Jeden etwas Anderes. „Das Individuum muss nach dem eigenen Bedürfnis die Religion in den Einzelheiten gestalten; sie darf nur in allgemeinen Umrissen und nicht mit Zwang ihm geboten werden und muss so elastisch sein, dass sie leicht der Besonderheit des Menschen entspricht.“

Die Grundlagen der Religion sind Gemüt und Gewissen. Das Gemüt als Sammelbegriff sittlicher Qualitäten betrifft Empfindungen der Lust und Unlust und die davon abhängenden Triebe, Begierden und Handlungen.

„Eine wahre Religion, die das Gemüt veredelt und die Leidenschaften bannt, im Glücke den Übermut, im Unglücke die Verzweiflung nicht erwachen lässt, die wesentlich dazu beiträgt, den Menschen temper [ausgeglichen] zu machen und mit Liebe und Tugend zu erfüllen, sichert der Hygiene, der wirklichen Beglückung des Menschen am besten Bestand.“

Beim Gewissen, unterscheidet man das persönliche und das moralische Gewissen. Ersteres gibt uns die Vorstellung von uns selbst, von unserer Person und von der Tätigkeit unseres Geistes. Das moralische Ge- 
wissen ist jene innere Stimme, die angibt, was an sich gut oder böse ist. Es vermittelt die Pflicht, das Gute zu tun und das Böse zu unterlassen. Das sittliche Gefühl als Grundpfeiler der Moral ist nur in seiner entwickelbaren Anlage angeboren. Es wird durch die Lebensverhältnisse, durch Erziehung, politische und soziale Einflüsse und durch die Umwelt in seiner Entwicklung beeinflusst. Es muss durch eine sich auf wahre Moral stützende Erziehung entwickelt werden.

Reich schließt das Kapitel der Moralischen Hygiene mit der Erkenntnis, dass Unwissenheit und Materialismus (übermäßige Selbstsucht und Herrschaft des Geldes) Krankheit und Elend erzeugen; Vernunft und Liebe jedoch sind die besten Mittel dagegen, die besten Maßnahmen der Gesundheitspflege.

\section{Die Soziale Hygiene}

Die Soziale Hygiene nimmt das Wohl der ganzen Bevölkerung wahr. Mit Hilfe der Statistik überblickt sie die Gemeinschaft in deren verschiedenen Zuständen und verfolgt die Erscheinungen des gesellschaftlichen Lebens. Dieses kann Krankheitsursache für den Einzelnen sein durch z. B. schlechte Gesetze und Einrichtungen, falsche Erziehungsgrundsätze, üble Moral, böse Beispiele, Übermut Einzelner, Elend, Kriege, verkehrte nationalökonomische Fakten, falsche Gerichtsbarkeit, unzureichende Gesundheitsbetreuung, mangelnde öffentliche Sicherheit [30]. Das körperliche und soziale Wohlergehen einer Bevölkerung wird auch sehr durch genügende Ernährung gefördert, denn je leichter ein Mensch sich seinen Unterhalt verschaffen kann, desto weniger hat er es notwendig, seine Lage durch unethische oder kriminelle Handlungen zu verbessern. Überall wo „Knechtschaft [Abhängigkeit] und Massenarmut innerhalb eines Gemeinwesens" herrschen, ist dessen Bestand durch entstehende rohe Gewalt und blinde Wut gefährdet. Schlechte gesellschaftliche Zustände können auch epidemische Krankheiten fördern, die ihrerseits die sozialen Verhältnisse noch verschlechtern können.

Will man also eine Bevölkerung glücklich und gesund erhalten, so sind viele Maßnahmen gleichzeitig notwendig, insbesondere eine geordnete Staatsverwaltung, gute Gesetze und Normen, Gerechtigkeit im Richteramte, Verminderung von Steuern und Abgaben, Verhütung der Unwissenheit der Bevölkerung durch guten Unterricht und Förderung von Wissenschaft und Kunst, Begünstigung gemeinnütziger Unternehmungen, Abschaffung der Standesvorrechte und des „Parasitentums“ [Korruption], Ehrlichkeit in den öffentlichen Geldgeschäften und überhaupt gutes Beispiel der in der Öffentlichkeit stehenden Personen. Länder, in denen dies fehlt und deren Herrschaft und Kampf der Parteien den Besitz in Frage stellen, vergiften die Moral und zeigen das Bild von sozialer Erkrankung wie geistige Störungen, Verbrechen, Selbstmord und Laster.
Eine wesentliche Ursache sozialer Gesundheit ist die Arbeit, die auf den drei Grundpfeilern Moral, Hygiene und Ökonomie beruht. Darum müssen die Unternehmer „barmherzig“ sein [sozial handeln] und [in Innungen] „zusammentreten, um ohne wirkliche Selbstschädigung das materielle und moralische Wohl der Arbeiter sicher zu stellen“. „Wo die Arbeit nicht ausreicht, tritt die Barmherzigkeit [soziale Fürsorge] ergänzend ein, wo die Arbeit Übermaß gewährt, tritt die Vernunft regulierend ein“. Dafür ist es unerlässlich, dass Arbeit und arbeitende Menschen geachtet werden und diese nicht nur als bloße Mittel und Werkzeuge für den Gewinn Anderer dienen. Die Arbeiter selbst „müssen sich assoziieren, Groll und Bitterkeit wider ihre Arbeitgeber fallen lassen“. Also „Moral und Ökonomie schließen bei Arbeitern und Arbeitgebern einen unauflöslichen Bund zum Zwecke, auf beiden Seiten den Grundsatz zur Geltung zu bringen ,Jedem das Seinige', um auf beiden Seiten Tugend und Glückseligkeit zu ermöglichen und zu fördern.“ Die Gemeinschaft kann durch freie Vereinigungen aller Beschäftigten, Hilfsvereine, Vorschusskassen und Sparbanken dazu beitragen. Und es „kommt die Hygiene und sagt den Arbeitgebern, bauet Häuser für Die, deren Fleiß euch Reichtum, Bildung, Gesundheit und Ehre gibt", ermöglicht ihnen ein ordentliches Leben, „erhellt ihren Geist, veredelt ihr Herz“, macht sie „selbstständig, kräftigt ihre Assoziation, erhaltet sie gesund“.

Jeder Mensch muss immer von seiner Arbeit leben können und der Lohn der Arbeit muss wenigstens hinreichend sein, den Unterhalt für ihn und seine Familie zu sichern. Die Höhe des Lohns hängt von der Ausbildung des Arbeiters, aber auch von äußeren Verhältnissen ab. Die Arbeit soll dem Arbeiter aber auch Freude bereiten, wozu auch die Möglichkeit beiträgt, in seiner Freizeit seinen Geist zu bilden. „Je mehr sich die Gesellschaft entwickelt, desto vollkommener wird die Arbeit, desto mehr wird sie vergeistigt, desto mehr endlich stellt sie den Menschen sicher."

Armut kann aber nicht nur die Folge mangelhaften Lohnes sein, sondern ist auch häufig genug das Endergebnis unpassender Anwendung eines allzu großen Lohnes mit der „Folge von Üppigkeit und Ausschweifung, von Überkultur und Überreizung“. Überhaupt ist die Art der Verwendung der materiellen Güter von hervorragender Wichtigkeit für die soziale Gesundheit, denn ein sehr großer Teil des Nationalvermögens wird von allen Klassen der Gesellschaft teils unproduktiv, teils zur Zerstörung der produktiven Kräfte verwendet.

Einfluss auf die soziale Gesundheit haben auch die soziologischen Bewegungen, also die Zahlen der Geburten und Todesfälle, der Eheschließungen und -scheidungen. Diese werden u.a. beeinflusst durch Moral, Stand der Aufklärung, Wohlstand, Beschäftigung, Konstitution, Verhältnis des Alters beider Geschlechter und auch vom Klima. Es muss also der natürliche Ausfall von Mitgliedern durch gesunde 
und kräftige Nachkommen gedeckt werden, die zu gesunden, vernünftigen und anständigen Menschen erzogen werden. Es ist daher Aufgabe der Sozialen Hygiene, durch Beeinflussung der Zeugenden für die Gesundheit der Gezeugten zu sorgen, somit die Fruchtbarkeit der Menschen normal zu erhalten, also sie weder über das Maß hinaus zu vermehren, noch auch künstlich zu beschränken. Dafür dient die Ehe als „Grundlage der Familie und dadurch derjenigen Anstalt [Institution, Einrichtung], worauf die Überlieferung aller menschlichen Sitte und Bildung beruht. Sie ist die Grundlage der Staaten, weil der Mensch in der Familie die sittlichen Eindrücke, die Gewöhnung an Zucht und Ordnung, und die Ehrfurcht vor der Autorität empfängt, ohne welche kein Staat bestehen kann.“ Dieser soll Ehen „zwischen Individuen mit ähnlichen Familienanlagen, Menschen mit wirklichem Kretinismus, mit Epilepsie und ähnlichen Leiden" sowie unter Verwandten verhindern. Er soll jedoch Sorge tragen, „dass Verbindungen zwischen vollkommen fremden, einander sogar in Nationalität verschiedenen Menschen zu Stande kommen" [10, 31]. Ein weiterer Zweck der Ehe ist das Einstehen der Ehepartner für einander. Durch die Förderung der legitimen Ehen in den arbeitenden Klassen wird die Säuglings- und Kindersterblichkeit verringert. In der Folge ergibt sich eine der wichtigsten sozial-hygienischen Maßnahmen, die Verpflichtung der Kinder und Minderjährigen zum Schulbesuch. Die Schule muss die notwendigen Kenntnisse und die bürgerlichen Tugenden vermitteln, sie darf jedoch nicht für die Interessen politischer und kirchlicher Parteien missbraucht werden.

Soziologische Bewegungen entstehen auch durch Migration und betreffen Auswanderungs- und Einwanderungsländer. Die Folgen der Auswanderung kann für manche Länder ein Gewinn, für andere ein Verlust sein. Die ausgewanderten Menschen aus nicht oder schlecht ausgebildeten Schichten werden in ihrer Heimat bald ersetzt. Dagegen hat die Auswanderung von beruflich und geistig fähigen Menschen eine größere Nachwirkung, da die zurückbleibende Gesellschaft meist nicht die Mittel besitzt, diese rasch $\mathrm{zu}$ ersetzen. Andererseits kann aber auch im Einwanderungsland die soziale Gesundheit beeinflusst werden. Wenn nämlich gesunde Menschen in genügender Zahl in ein Land einwandern, „dessen Bewohner in Entartung begriffen sind" [31], so ist gesunder Nachwuchs und ein verlängertes Leben der ganzen Bevölkerung zu erwarten.

\section{Die Diätetische Hygiene}

Die Diätetische Hygiene oder Diätetik kümmert sich um das körperliche Wohl des Einzelnen, um die Normalerhaltung aller Verrichtungen, die außerhalb der bewussten Gehirntätigkeiten liegen. Das Wohlsein des Menschen wird davon bestimmt, wie er die leiblichen Bedürfnisse befriedigt und wie er von seinen Organen
Gebrauch macht. Für die Erhaltung der Gesundheit ist also die richtige Verwendung der Nahrungs- und Genussmittel, der Kleidung, der Körperübungen und der Körperpflege, das Maßhalten in Schlafen und Wachsein und in den Aktivitäten der Fortpflanzung wichtig. Auch einen großen Einfluss können manche der von einem großen Teil der Bevölkerungen nicht oder nur schwierig zu beeinflussenden Umstände haben, wie insbesondere die Wohnverhältnisse, aber auch die Einflüsse von Atmosphäre, Licht, Boden, Klima und die Veränderungen durch Wechsel des Aufenthaltsortes. Für die Erhaltung der Gesundheit bemüht sich die Diätetische Hygiene, Maßnahmen für positiven Einfluss auf diese bestimmenden Faktoren zu finden und anzuwenden.

Ihr weitergehendes Ziel ist nicht nur die Erhaltung der Gesundheit, sondern auch ihre Ausdehnung auf ein möglichst hohes Alter. Die Quelle eines langen Lebens ist die gute Konstitution des Menschen, die einerseits von seiner körperlichen Anlage abhängt, welche ein Ergebnis des Zustandes seiner Erzeuger ist. Andererseits sind auch die Mäßigkeit und die Gleichmäßigkeit in sittlicher und leiblicher Beziehung, sowohl hinsichtlich der Nahrung, des Beischlafs wie aber auch jeder anderen Sache notwendig. Reich meint, dass mit solchem gutem Verhalten das lange Leben erblich wird und dass alle Völker mit langer Lebensdauer diese der genauen Befolgung diätetischer Vorschriften verdanken.

Nach Reichs Auffassung beruht die Diätetische Hygiene auf dem Einsatz von Vernunft und Vorsicht, von Übung und Mäßigung, somit gründet sich wahre Diätetik auf Moral und leiblicher Erstarkung. Sie kann nur in kleinen Schritten vorankommen, weil sie nur durch Beraten und Belehren, nicht durch Zwang wirkt. Sie muss sich vor allem mit der Erziehung verbünden, weil nur diese der Diätetik Eingang, Geltung und Herrschaft sichert. Gewiss ist der Erzieher immer der beste Verkünder und Sachwalter der Diätetik, weil er, mehr als andere, den Menschen und zumal die Jugend beeinflusst.

Der Arzt soll beim Kranken durch diätetische Heilmethoden Gesundung erreichen und dem Gesunden durch Empfehlung entsprechender Maßnahmen der Diätetischen Hygiene Wohlbefinden und Lebensfreunde verschaffen.

Bei den einzelnen Maßnahmen für eine gute Diätetische Hygiene steht die Ernährung für Reich an erster Stelle, denn ,alles dreht sich ums Futter“. Die Diät muss alle im Stoffwechsel verloren gegangenen Stoffe ersetzen und auch noch die dem Organismus fehlenden Substanzen einbringen, aber auch die im Übermaß vorhandenen verringern. Je naturgemäßer die Nahrung ist, desto gewisser hilft sie, die Gesundheit zu erhalten, die Krankheit zu verhüten. Die Auswahl der Nahrungsmittel wird durch individuelle und äußere Verhältnisse bestimmt, von Lebensalter, Sitten, Gebräuchen, Vorurteilen und vermeintlichen Bedürfnissen, von äußeren Einflüssen wie Klima und Be- 
schäftigung. Reich bespricht die verschiedenen Nahrungs- und Genussmittel und ihren Gebrauch in den Lebensaltern.

Bei der Diätetik des Körpers kommt der Pflege der Haut eine große Bedeutung zu, denn sie dient unter der jeweils geeigneten Kleidung dem äußeren Schutz des Körpers. Bei ihrer Reinigung spielt das Bad eine unerlässliche Rolle. Es nimmt nicht nur den Schmutz von der Haut, es wirkt auch positiv auf die darunterliegenden Muskeln, Blutgefäße und Hautnerven. Die Haut wird auch von der Kosmetik mehr oder weniger betroffen. Die „hygienische Kosmetik“ soll empfehlen, sich mit klarem, frischem Wasser vom Schmutz der Geschäfte, des Tuns und des Nichtstuns zu reinigen und keine wohlriechenden Wässer, Pomaden, Salben und Öle zu verwenden. Die „weltliche Kosmetik“ sorgt durch gepflegtes Äußeres und die der jeweiligen Situation angepasste Kleidung und entsprechenden Schmuck für die soziale Akzeptanz.

Eine unerlässliche Voraussetzung gesundheitsgemäßer Entwicklung des Leibes ist die Gymnastik. Ihre Vernachlässigung hat nicht selten Siechtum und Leiden zur Folge. Systematische Leibesübungen müssen Gegenstand der Erziehung sein. Reich bespricht die Vor- und Nachteile des Turnens, Reitens, Tanzens, Laufens, Marschierens, Schwimmens und auch von körperlich getätigten Spielen.

Er legt Wert auf die „Harmonie der Sinneswerkzeuge" als Voraussetzung voller Gesundheit. Diese bedürfen schon in frühester Jugend der Pflege, denn je richtiger die sinnlichen Wahrnehmungen sind, desto mehr besteht die Möglichkeit korrekter Schlüsse und einer normalen Lebensanschauung. Für die Entwicklung und Pflege der Sinne werden verschiedene Maßnahmen angegeben.

Als hygienische Regeln für den lebensnotwendigen Schlaf sagt Reich: man soll in geräumigen, kühlen oder mäßig warmen, gut ventilierten, trockenen Räumen ohne grelles Licht schlafen, vor dem Schlafengehen esse man nicht zu viel, entleere Harn und Stuhl und vermeide, sich durch Leidenschaften und Gefühle stark aufzuregen. Jedoch möge „wer ausgewachsen ist, den erforderlichen Überschuss von Kraft hat und ohne künstlich angeregt werden $\mathrm{zu}$ müssen, den wahren Drang zur Begattung fühlt, das Geschäft der Fortpflanzung besorgen“.

Der Wohnsituation des 19. Jahrhunderts entsprechend sieht Reich gesundheitsgemäße Wohnungen für Arme und Arbeiter als ersten Schritt aus dem Elend und den Umbau ganzer Stadtteile als beste Möglichkeit, die allgemeine Gesundheit zu verbessern. Dicht bevölkerte Wohnorte haben hohe Krankheits- und Sterblichkeitsverhältnisse und in weiterer Folge eine höhere Kriminalität. „Ungesunde, unter den schädlichen Einflüssen der Dunkelheit, der verdorbenen Luft, der Feuchtigkeit, der Kälte, des Hungers lebende Bevölkerungen verharren in ungeregelten Instinkten und in Leidenschaften. Die Macht der Erziehung bleibt eine ihnen unbekannte, eine gegenüber ihnen unwirksame Macht.“ Zu Verlängerung des Lebens und Verbesserung der Gesundheit wird die Wohnung beitragen, wenn sie in guter Gegend, auf gutem Boden steht, den nötigen Raum bietet, trocken ist, Licht und Luft durch große, gut schließende Fenster einlässt und frei ist von übelriechenden Ausströmungen [wegen noch fehlender Kanalisation].

Der Mensch ist ein Produkt des Klimas, von dem er abhängt. Er muss sich ihm anpassen und ihm auch Widerstand leisten, um die Gesundheit zu bewahren. Aber „auch die Moral wird vom Klima bedingt.“

Jeder Mensch lebt innerhalb seiner klimatischen Verhältnisse, denen er mehr oder weniger durch wechselndes Wetter ausgesetzt ist. Im einheimischen Klima beeinflusst es ihn umso weniger je gesünder, elastischer und abgehärteter er ist. Im Allgemeinen ist mildes Klima für den Menschen am förderlichsten. Lebt er in ihm fremden Klimazonen, so hängen Gesundheit und Wohlergehen davon ab, wie sehr er Fähigkeit und Willen besitzt, sich zu akklimatisieren. Er „muss strenge nach der Hygiene leben und sich selbst mit eiserner Gewalt beherrschen, muss vorsichtig und klug sein“. So wurden die verheerenden Gefahren des tropischen Klimas, die „aus dem Miasma der Sümpfe entspringenden Wechselfieber“ [Malaria], überwunden, nachdem „man angefangen hatte, sich hygienisch zu bilden und die Hygiene auszuüben“. „Es genügt aber nicht, vermittelst der Hygiene und der Bildung dem Klima Widerstand zu leisten; es ist auch nötig, das Klima öfters durch Reisen zu wechseln“.

\section{Die Polizeiliche Hygiene}

Die Polizeiliche Hygiene oder Gesundheitspolizei (Staats-Hygiene) bemüht sich um das Wohlbefinden der ganzen Bevölkerung und hat die Gesundheitsbehörde als vollziehendes Organ. Ihre Aufgabe ist die „Aufsuchung und Zerstörung oder Beseitigung der Krankheits-Ursachen und die Anordnung der Vorschriften und Regeln, deren Beachtung die unmittelbar leibliche Gesundheit des Volkes sichert". [Dies müssen wir heute durch das seelische und soziale Wohlbefinden ergänzen, WHO, 1946.] Die Gesundheitspolizei hat sich um die Verhinderung von Verfälschungen der Nahrungs- und Genussmittel und um jene Institute zu kümmern, die den Zwecken der sanitären Verhältnisse der Wohnsitze, Schulen, Kasernen, Gefängnisse, Fabriken, Lager und Reinigungsanstalten wie auch der Verhütung und Bekämpfung endemischer und epidemischer Krankheiten dienen.

Die Polizeiliche Hygiene muss „nicht predigen, nicht bitten, nicht vorschlagen, unterbreiten, unmaßgeblich glauben: sondern befehlen wird sie auf Grund wissenschaftlicher Überzeugung“. Denn trotz der Achtung der bürgerlichen Freiheit des Einzelnen und seiner Ablehnung einer Einmischung des Staates in private Angelegenheiten muss man jedoch "Jedermann nötigen, sich dem Gesetze der Gesundheit 
zu unterwerfen, Maßregeln der Gesundheitspflege zu vollziehen und das Amt der Gesundheit zu respektieren“. Die Polizeiliche Hygiene fordert daher „Gehorsam wegen des allgemeinen Besten, wegen der allgemeinen Glückseligkeit; sie rügt den Saumseligen, sie bestraft den Übertreter ihrer Vorschriften“, weil „er seine und seiner Mitbürger Gesundheit gefährdet“. Es ist also kein Widerspruch, wenn der Staat „in den Topf, in das Bett, in den Abtritt [Abort] des Staatsbürgers seine Nase steckt“. Der Staat fragt dabei nicht nach dessen Glauben und Überzeugung, er will nur das Verhalten jedes Staatsbürgers in gesundheitlicher Hinsicht reglementieren.

Die Hygiene anerkennt also nicht eine utopische absolute Freiheit, sondern nur jene relative Freiheit, durch die „der Einzelne befähigt wird, das Gute zu vollbringen, das Böse, das Schädliche zu erkennen und zu beseitigen“. Diese „Selbsthilfe“ genügt allein ebenso wenig wie eine ausschließliche „Staatshilfe“ für die Erreichung der Ziele der Gesundheitspolizei. Diese soll die Anleitung und Führung geben, denn wenn sie allein tätig ist, „kommt nur zu leicht ein bürokratischer Geist in die Maschine und nicht mehr das Heil der Menschen ist der rote Faden, sondern das Vorurteil, der Dünkel und die Borniertheit des Schreibertums ist es."

Die ausübende, insbesondere die Polizeiliche Hygiene muss, da ihr schlimmster Gegner das Elend ist, mit Barmherzigkeit und Menschenfreundlichkeit einher gehen und „immer und überall die falschen Folgerungen einseitiger und gemütloser Nationalökonomen paralysieren“. Es ist also „dringend geboten, der falschen und unsittlichen Ökonomie kräftigst entgegen zu arbeiten, die Barmherzigkeit und Humanität anzufachen und durch Förderung des Vereinswesens [Hilfsvereine, Vorsorgekassen, Sparbanken] und sonstiger die allgemeine Wohlfahrt bezweckender Institute das Sinken Einzelner zu verhüten, bereits Gesunkene dem normalen Leben wieder zu geben.“

\section{Der Rat der Wohlfahrt}

Im Bereich der Polizeilichen Hygiene konzentriert Reich seine „Ansichten und Wünsche“ darauf, dass „Alles, was in die Breite der Gesundheit, Erziehung, öffentlichen Wohltätigkeit und Sicherheit fällt, vor das Forum eines Rates der Wohlfahrt gehöre“. Dieser Wohlfahrtsrat gliedert sich in einen „Rat der Gesundheit“, einen „Rat der Erziehung“ und einen „Rat der Sicherheit (Polizei und öffentliche Hilfe)“. Alle drei Räte bestehen aus einem „gesetzgebenden Teil“ und einem „vollziehenden Teil“. Die Mitglieder des gesetzgebenden Teils sollen vom Volk gewählt und dem Rat zu einem Drittel ständig, zu zwei Dritteln wechselnd angehören. Sie sollen „Hygieniker, Pädagogen, Moralisten, Naturforscher, Ärzte, Apotheker, Veterinäre, Kameralisten, Polizisten, Techniker und höher Gebildete ohne bestimmtes Fach sein“. Die selbst gewählten Vorsitzenden sollen jährlich wechseln. Solche
Wohlfahrtsräte sollen ihre Tätigkeit als Departementalrat, als Provinzialrat und für den Gesamtstaat als Generalrat ausüben. Sie müssen frei von Einflüssen aus Parteien, Politik, Regierung und Kirche sein, denn „der Rat der Wohlfahrt kann eine Autorität über sich nicht dulden“.

„Nur der Rat der Wohlfahrt soll, nach Anhörung der öffentlichen Meinung, Gesetze der Gesundheit beschließen und ausführen." Diese müssen alles umfassen, was sich auf die Beseitigung oder Verhinderung der Wirkung von Krankheitsursachen bezieht, somit primär auf alle physischen Verhältnisse, die auf den Menschen einwirken. „Nicht Vorschriften des Verhaltens, sondern Vorschriften eines die Gesamtheit der Bürger umfassenden hygienischen, oder speziell bezeichnet: nosophthorischen [nosos=Krankheit, phthora=Zerstörung], Regiments sind die Gesetze der Gesundheit.“ Sie müssen „dem Zustand des Staates wie dem Geiste des Volkes, für welche sie gegeben werden, angemessen sein“. Die Gesundheitsgesetze sollen eine klare, jedes Missverständnis ausschließende Sprache führen. Wenn diese Gesetze alldem entsprechen, „ist man berechtigt, den Übertreter, den Verletzer derselben zu bestrafen“.

Aber „das strengste Gesetz der Gesundheit und dessen exakteste Durchführung beseitigen nur Symptome und dies nur für den Augenblick; ohne eine mit der Moral harmonisch vereinigte Ökonomie, ohne Beseitigung des Elends und Tilgung der übermäßigen Selbstsucht, ohne naturgemäße Bildung des Geistes und Veredelung des Herzens hat das Gesetz der Gesundheit keine Basis“.

Den unter den Mitgliedern der Wohlfahrtsräte an erster Stelle genannten Hygieniker beschreibt Reich als „Mann, der auf Grund philologischer, mathematischer, philosophischer, historischer und literarischer Bildung die Naturwissenschaften, die Medizin und die politisch moralischen Wissenschaften studierte und, auf dieser Basis stehend, die gesamte Hygiene sich zu eigen machte. Es kann die Hygiene in ihrem ganzen Umfang und als Philosophie, Wissenschaft und Kunst des gesunden Lebens, der Erhaltung der Gesundheit und der Abwendung wie Zerstörung der Krankheitsursachen, nur auf der bezeichneten Basis studiert und begriffen werden, und es wird in allen die Wohlfahrt und Gesundheit betreffenden allgemeinen Fragen nicht der Arzt als solcher, der Moralist als solcher, der Techniker als solcher, sondern nur der Hygieniker als solcher kompetent sein."

Zur Behebung des Mangels einer universitären Ausbildung von Hygienikern, die sich ja bisher ihr Wissen selbst aneignen mussten, fordert Reich die Errichtung von Lehrstühlen für moralische, soziale und diätetische Hygiene und solche für polizeiliche Hygiene an den Universitäten. Daneben sind für das Studium der Wohlfahrt auch die von den Universitäten angebotenen Unterrichtsfächer der Medizin, der Pädagogik und der Politisch-moralischen Wissenschaften, also der Moral, der Sozialwissenschaft, der Politik und 
der Polizei notwendig. „Für ein erfolgreiches Studium der Medizin und für das genaue Verständnis der ganzen Hygiene“ ist nämlich eine geläuterte, abseits aller Systeme gelegene Philosophie von besonderer Wichtigkeit. Beide können weder in der Lehre noch in der Ausübung bei den gefundenen Tatsachen stehen bleiben, denn sie müssen „dieses Gewirr trügerischer und eingebildeter Verbindungen von Ursache und Wirkung“ entwirren und „von denen trennen, welche wirklich den physischen und moralischen Gesetzen der Natur gemäß sind“. Hierfür bedürfen Medizin allgemein und Hygiene nicht der Schulphilosophie, „sondern jener reinen und, weil systemlosen, darum naturgemäßen Philosophie, die aus dem unbefangenen Studium unbestreitbarer Tatsachen quillt".

Die Universitäten sollen „unabhängig vom Staat, von der Kirche und den herrschenden Parteien, Rotten, Sekten“ sein wie auch von „Geckentum und Vorurteilen der Gesellschaft“ und auch „frei von Kasten- und Zunftgeist“. Es erscheint zweckmäßig, dass neben den Staats-Universitäten auch von einzelnen Staatsbürgern unterstützte freie Universitäten gegründet werden, die alle aber nicht in Fakultäten gegliedert sind. Es seien nur eine einzige Art von Professoren mit einem für ein anständiges Leben reichenden Gehalt zu bestellen, und zwar von der Universität selbst und nicht vom Staat. Für ,jeden Zweig des menschlichen Wissens und Könnens" genüge im allgemeinen ein Professor und daneben eine nicht beschränkte Zahl von Dozenten. Die Kollegiengeldzahlungen und die Prüfungen würden für Studenten entfallen. Die „Doctors-Würde“ soll beibehalten werden, jedoch wegen der geforderten Aufhebung der Fakultäten nur von der Universität selbst und nur für wissenschaftliche Verdienste erteilt werden. Niemals aber solle man das Doktorat aus eigenem Bestreben in einem Studium erwerben können, sodass „kein Praktiker genötigt werde zu promovieren“. Die Venia legendi könne jedem, der sich durch wissenschaftliche Arbeiten bekannt gemacht hat, ohne Weiteres erteilt werden.

Nach dieser Erörterung seiner Forderungen für die Ausbildung der Hygieniker und für die Universitäten allgemein kommt Reich zu den Aufgaben der politischen Hygiene, zum Einsatz der Gesundheitspolizei in ihren Teilgebieten.

Die Gesundheitspolizei der Nahrungs- und Genussmittel muss verhüten, dass die Konsumenten durch verdorbene oder vergiftete Nahrung krank werden oder sterben. In der Gesellschaft wird aus „Gewinnsucht, Gemeinheit, Herzens-Härtigkeit der Einen und Not der Anderen“ deren Gesundheit durch die Abgabe schlechter oder unzureichender Nahrung gefährdet. „Solange es Arme und diesen gegenüber Feigheit und Nichtswürdigkeit unter den besser Gestellten gibt, muss die bürgerliche Gemeinschaft ihre schützende Hand über die Unglücklichen halten“. Reich nimmt die Gesamtheit der Bürger in die Pflicht, „Vorräte unverfälschter, guter Nahrungsmittel zu sammeln und in der Zeit der Not an alle Bedürftigen zu verteilen“.
Gerade in Notzeiten stehen „dem leider nicht sporadischen, gewissenlosen Betrug Türen und Tore offen; gerade in diesen Zeiten wird des Leibes Bedarf, und insbesondere jener der Armen, am meisten verfälscht. Der Arme hat nicht die Mittel, gute und unverfälschte Nahrung sich zu verschaffen; es muss somit durch die öffentliche Autorität reichlich ihm geboten werden.“ Damit aber nicht auch einzelne ihrer Organe aus Gewinnsucht Fälschungen vornehmen, „soll eine aus Unparteiischen, aber großenteils Sachverständigen zusammengesetzte Behörde über die Güte der Vorräte wachen, und zumal vor jeder öffentlichen Austeilung von Lebensmitteln dieselben genau auf Echtheit prüfen“.

Gerade pflanzliche Speisen sind für Missbrauch durch „Gemeinheit und Gewinnsucht" geeignet. „Man kann behaupten, dass die Fabrikanten, weil mit Kenntnissen aus der Chemie mehr oder weniger stark geschwängert, diese Kenntnisse und ihre natürlichen Geschicklichkeiten nicht zum Wohle, sondern zum Verderben der Menschen anwenden“. Den Unterschied zwischen Betrug und Gesundheitsgefährdung der Bevölkerung macht Reich an einem Beispiel deutlich. Wird an irgendjemandem guter Weißwein statt echter Champagner verkauft, so wäre dies Betrug, aber ohne irgendwelchen Nachteil für die Gesundheit; wenn aber dem Brot Kupfervitriol oder der Schokolade Quecksilberoxyd zugesetzt werden, dann ist dies ein schwerer Angriff auf Leben und Gesundheit der Bevölkerung.

Reich bespricht die Vor- und Nachteile der verschiedenen Gruppen von Nahrungs- und Genussmitteln und von Geschirren.

\section{Gesundheitspolizei der Institute und der Wohnsitze}

Das Herz zieht sich Reich krampfhaft zusammen, wenn er „Anleitung dazu geben soll, die Menschen strotzend von Gesundheit zu machen, damit sie mehr Kraft" haben, als Soldaten einander abzuschlachten oder als Arbeiter sich „im Interesse der Gewinnsucht des Fabrikanten von der Maschine zermalmen und Weib und Kinder in Hunger, in Elend zurück zu lassen“.

„Harte, schwere Kämpfe verursachte das Eindringen der Hygiene in das Labyrinth der Institute; aber Wohlsein der Menschen war die letzte Folge“. Denn „von dem Geiste der Nächstenliebe durchdrungen, wünschen wir, dass alle Institute den Zwecken der Liebe, der Barmherzigkeit und des allgemeinen Nutzens dienen“. Weil nun Menschen, freiwillig oder unfreiwillig, in solchen Anstalten etwas zu ihrem Nutzen suchen, „darum werden alle Institute von dem SpäherAuge der Gesundheitspolizei beobachtet und kontrolliert".

Unter „Gesundheitspolizei der Wohnsitze“ erörtert Reich die hygienischen Bedürfnisse und Maßnahmen für die Menschen in Häusern, „welche sie mit ver- 
schiedenen Namen benennen und verschiedenen guten und schlimmen, vernünftigen und tollen Zwecken widmen. Diese Häuser und all' das Volk und all' die Sachen, welche dazu gehören, die Namen, die Zahlen, die Titel, die Kleider und die Hantierungen, dies zusammen genommen macht das Institut aus."

Von der Besprechung der Hygiene der Wohnsitze sei nur die in den 1870er-Jahren heftig diskutierte „Entwässerung und Fortschaffung der Auswurfstoffe“ aufgegriffen. Reich schloss sich der Meinung an, dass die Entfernung der Exkremente über Wasserklosetts und Kanäle sehr verderblich ist. Es sei nämlich unvermeidlich, dass durch deren Einleitung in Bäche und Flüsse, selbst wenn sie stark wie der Mississippi wären, diese verpesten und in der Folge das Grundwasser verunreinigen. Besonders bei Überschwemmungen treten die bekannten Darminfektionen auf. Es ist deswegen der Entfernung der Exkremente auf trockenem Weg, sei es pneumatisch oder durch Abfuhr, vorzuziehen.

\section{Institute von Kultus und Unterricht}

Die „Institute des Kultus und des Unterrichts“ haben teils allgemeine, teils ihre eigenen, speziellen Probleme.

Wenn die Kirche „das Feuer der Liebe entzündet in den Herzen alles Volkes und erstickt den Schwefelbrand des Hasses und der Selbstsucht, des Neides und des Geizes“, dann bewirkt sie „Gesundheit der Herzen und Heil und sie beglückt“. Es ist gleichgültig, ob „der Halbmond oder das Kreuzenzeichen sie schmücke, ob Moses darin walte oder Buddha oder Brahma“. „Aber unterstützt muss werden das Gute und Edle durch wohl gelungenen Bau der Kirche, durch Wärme, Licht und trockene Luft, denn wer mit Freude lauschen soll den Worten des Verkündigers der Liebe, des Spenders des Trostes, des Mahners der Pflichtvergessenen, muss auch leiblich sich wohl fühlen in den Räumen des Friedens, der Ruhe, der Erbauung.“

Der Rat der Wohlfahrt hat „die Pflicht, der Ausführung gemeinschädlicher Anordnungen und Gebräuche entgegen zu treten: er darf nicht gestatten, dass zumal während des Herrschens einer Seuche das Volk stundenlang auf kalten Steinen kniet“ oder Wallfahrten durchführt, er muss Kasteiungen und schädliche Bußübungen untersagen.

Reich, der in seinen Publikationen sehr oft seine antiklerikale Einstellung, zum Teil auch recht aggressiv, deutlich macht, stellt aus gesundheitspolizeilicher Sicht die Frage, ob es Klöster geben soll. Er bezeichnet diese jedoch objektiv als „Orte, wo Unglückliche, Lebensmüde, Gebrochene Ruhe und Erhebung finden; aber diese Orte sollen nicht die Ehe ausschließen, nicht Schauplätze von Übungen sein, welche die Gesundheit zerstören und das Leben in Frage stellen; sie sollen den Menschen nicht für Lebenszeit binden und stets nützlichen Dingen dienen, z. B. dem Unterrichte der Jugend, der Krankenpflege, der Besserung
Verwahrloster, der Erziehung von Verbrechern, der Tilgung von Not und Elend etc.". Reich ist durchaus nicht für die sofortige Schließung der Klöster, bleibt aber seiner Einstellung treu, wenn er für deren allmähliches Erlöschen durch das Vermeiden der Aufnahme neuer Mönche und Nonnen ist. Andererseits meint er aber, es müsse „Stätten geben, wo der Unglückliche Zuflucht findet, Ruhe des Gemüts, den ersehnten Frieden und das liebe Brot: Klöster der Kirche der Menschheit“. Letztere beschreibt er aber nicht.

Das Institut des Unterrichts ist ein wichtiger Teil der Polizeilichen Hygiene, denn eine gute Bildung ist, wie Reich in seinen Schriften sehr oft betonte, eine ganz wesentliche Grundlage für erfolgreiche Hygiene. Es ist interessant, dass er dabei eine besondere Aufmerksamkeit auf die Belastbarkeit der Schüler lenkt. „Der durch Überhäufung beim Lernen gelähmte Geist kann nämlich das Dargebotene nicht verarbeiten, und dies dient also nur dazu, dass die Knaben dem Examen genügen können und hierauf das Unverarbeitete wieder vergessen. Gleichzeitig werden aber solche mit Arbeit überhäufte Schüler sowohl der Natur als auch ihrer Familie entfremdet und überdies erfolgt in späterer Zeit eine Abstumpfung“ als Folge der Überreizung in der Jugend. Aber noch rascher leidet die körperliche Ausbildung durch das lange Sitzen in der Schule und bei den Hausaufgaben. Die Pädagogen überbürden oft die Schüler mit einer „Last großenteils nutzloser Gedächtnis-Sachen“. Deswegen ist es „sehr notwendig, die Zahl der Schulstunden zu beschränken, die Hausaufgaben abzuschaffen, Nachmittags-Unterricht nicht zu erteilen, und die Schüler, außer Gymnastik im vollen Umfange“, auch Arbeiten in Garten und Werkstatt verrichten zu lassen. Denn so können in der Jugend auch gewisse, dem praktischen Leben sehr zu Statten kommende Kunstfertigkeiten und handwerkliche Fähigkeiten ausgebildet werden. „Der Gesundheit gemäß wäre die Scheidung des Schuljahres in drei Schulquartale und ein Ferienquartal.“

„Wenn der Lehrer wirklich ein geistiger Vater des Kindes sein und dieses auch sittlich leiten soll, darf er den jugendlichen Geist nicht in die Fesseln trockener Kenntnisse und unfruchtbarer Regeln schlagen, sondern muss, da er dem Schüler mit dem erforderlichen Spielraum des Geistes zugleich den unmittelbaren Verkehr mit der Natur gestattet, Geist und Herz und Leib mit einem Male pflegen“. Je weniger das Kind von Kenntnissen erdrückt wird, desto leichter wird erreicht, dass aus dem Herzen des Kindes alle Laster und zerstörenden Einflüsse verbannt werden, dass sein Gedächtnis gestärkt, sein Urteil befestigt und seine Einbildungskraft vergrößert werden.

Für den Unterricht in Schulen gibt Reich Hinweise auf Bau und Einrichtung der Schulgebäude, wobei die „Subsellien“ [Sitzbänke], der umfangreichen damaligen Literatur entsprechend, ausführlich behandelt werden. 


\section{Krankenhäuser und soziale Einrichtungen}

„Leider erzeugen Unvernunft, Lieblosigkeit und mechanische Verhältnisse eine Legion von Übeln, physischer und moralischer Art, machen unzählige Menschen hilflos und zum Gegenstande des Mitleids“. Da nun „der Mensch in der größeren Mehrzahl der Fälle nur sehr beschränkt dasteht“, werden „öffentliche Orte benötigt, wo Unglückliche und Leidende Aufnahme, Hilfe, Heilung der Leiden finden, oder wo die Barmherzigkeit das Scheiden ihnen leicht macht und die Überreste der ewigen Ruhe teilhaftig werden lässt: Hospitäler“.

Krankenhäuser sollte es überall geben und „in der Tat sollte die Gemeinschaft aller Bürger möglichst viele Hospitäler gründen und reiche Private sollten, nicht um bewundert und in Zeitungen gepriesen zu werden, sondern aus wahrer Menschenliebe das Gleiche tun“. Die Krankenhäuser müssen, ohne nach Vermögen, Herkunft, Nationalität u.s.w. zu fragen, jedem Bedürftigen offenstehen. Die angebotene Krankenpflege muss freiwillig sein, denn es ist gewiss, „dass der Krankenpfleger, welcher sein Amt des Brotes wegen verrichtet und nicht aus reiner Nächstenliebe dazu geführt wurde, in der Mehrzahl der Fälle der Menschheit keinen erheblichen Nutzen bringen werde. Der Rat der Wohlfahrt tut danach am besten daran, Philanthropen zur Krankenpflege aufzufordern, und nur jene Posten, welche Berührung mit den Kranken nicht erheischen, durch menschliche Maschinen zu ersetzen."

Alle Krankenhäuser eines politischen Gebietes sollen dem Gesundheitsrat dieser Region unterstehen. Dieser wird für deren Errichtung außerhalb von Städten und Dörfern Plätze mit gutem Boden in der Nähe von Gehölzen bevorzugen. Eine Versorgung mit gutem Quellwasser ist notwendig, jedoch soll das Spital nicht in unmittelbarer Nähe von Gewässern stehen, was eine Gefahr für die Gesundheit wäre; wie ja aus der Geschichte der Cholera zur Genüge bekannt ist. So ist auch die Einleitung der „Unreinigkeiten eines Krankenhauses“ in ein Gewässer verboten.

Danach stellt sich die Frage nach der Größe eines Krankenhauses. Am besten besteht es aus einer Anzahl kleiner Häuser, die nicht mehr als eine Treppe hoch sind. Jedes hat bis zu vier Zimmer, deren jedes mit höchstens vier Kranken belegt wird. Diese kleinen Häuser müssen getrennt mitten in einem Garten stehen. Ein solches Pavillon-System vereinigt die gesundheitlichen Qualitäten einzelner kleiner Spitäler mit den ökonomischen und administrativen Vorteilen von großen Krankenhäusern.

„Ein Gegenstand, welcher der größten Fürsorge von Seite der Polizei der Gesundheit bedarf, ist die innere Einrichtung der Hospitäler“. Das Hauptaugenmerk ist auf gute Reinig- und Desinfizierbarkeit, Vermeidung von Staub und üblen Gerüchen und guter Durchlüftung zu legen.
„Leider sind Krankenhäuser häufig Brutstätten endemischer Krankheiten und tragen zur Verbreitung herrschender Epidemien sehr wesentlich bei“. Etliche Menschen, die mit irgendeinem leichten Übel aufgenommen worden sind, verlassen die Spitäler als Leichen oder als von einem im Spital erworbenen Übel wieder Genesene. Als Ursachen gelten Feuchtigkeit und mangelhafte Lüftung der Räume, Unsauberkeit, Gerüche der Leibstühle und Aborte, schlimme Nachbarschaft der Kranken. „Im Großen und Ganzen lässt sich sagen, dass außer der Geschicklichkeit der Ärzte und der Gewissenhaftigkeit der Krankenwärter, die Hygiene es ist, welche die Sterblichkeit der Spitäler herabsetzt; je besser also ein Krankenhaus gebaut, eingerichtet, gehalten wird, desto geringer muss darin die Mortalität [recte: Letalität] sein“.

In der damaligen Diskussion, ob Findel-Häuser notwendige oder sogar schädliche Institutionen für die Unterbringung von Findelkindern [ausgesetzte Säuglinge und Waisenkinder] sind, lehnt Reich diese $\mathrm{ab}$ und wünscht die Errichtung von Findel-Kolonien „unter Leitung der Wohlfahrtsbehörde und unter spezieller Aufsicht eines Hygienischen Direktors“. Diese Kolonien sollen „in einer durchaus gesundheitsgemäßen Gegend mit gutem Trinkwasser und Nadelholzwäldern sich befinden, und wohlwollende Familien wie einzelne Frauen herbeiziehen, die gegen Überlassung von Haus, Garten und Feld der Erziehung der Findelkinder" sich annehmen. Natürlich müssten in diesen Kolonien Schulen u.s.w. bestehen, so dass den Kindern außer körperlicher Gesundheit und sittlicher Erziehung auch eine Geistesbildung sicher wäre. Solche Kolonien würden aus den Kindern brave, fleißige Menschen erziehen und nicht die Unzucht fördern, wie sie von den Findel-Häusern jener Zeit bekannt war.

„Die Hygiene der Entbindungs-Häuser ist im Großen und im Ganzen mit der Hygiene der Krankenhäuser gleich bedeutend“. Die Sterblichkeit ist unter gleichen hygienischen Verhältnissen dort am geringsten, wo die Räume mit gesundester Luft versorgt und nicht überfüllt sind und sich die kranken Wöchnerinnen streng gesondert aufhalten. Die EntbindungsHäuser sollen außerhalb der Städte und im PavillonSystem errichtet und nicht mit Spitälern oder FindelHäusern kombiniert werden.

\section{Gefängnisse}

Gefängnisse als Institute der Bestrafung durch Entzug der Freiheit und der bürgerlichen Rechte dienen aber auch der Erziehung und Besserung. „Da es nicht der Zweck humaner Gesetzgebung sein kann, den Menschen zu martern und Vergeltung an dem Unglücklichen zu üben, muss das Gefängnis durch Bau und Einrichtung geeignet sein, die leibliche Verfassung des Verbrechers, des sittlich und meistens physisch Kranken zu bessern; denn erst ein leiblich normaler Mensch ist moralischer Besserung und Vervollkomm- 
nung fähig. Gefängnisse, die wirklich Nutzen gewähren sollen, müssen Erziehungshäuser und Hospitäler mit einem Male [zugleich] sein. Die Gefängnisse alten Schlages sind Folterkammern, deren Wirkung vervollständigt wird durch die Vorurteile der Bevölkerung wider die aus der Haft entlassenen Bestraften.“

In jedem Gefängnis müssen den Gefangenen Licht, gute Luft und genügend Wasser zum Trinken und Waschen und genügende Ernährung zur Verfügung stehen, die Räume müssen frei von Feuchtigkeit, üblen Gerüchen, Unreinlichkeit, Hitze und Kälte sein. Da das hierfür günstige Pavillonsystem für Zellen-Gefängnisse nicht möglich ist, sollen diese, wie für Spitäler angegeben, auf freiem Felde erbaut werden. Geeignet sind Gefangenen-Häuser mit höchstens zwei Stockwerken und nicht mehr als zweihundert Insassen. Da die Fenster stets nur ins Freie und nicht in den Hof gerichtet sein dürfen, ist den Gefangenen das beliebige Öffnen zu gestatten.

„Die Beschäftigung der Gefangenen übt mittelbar wie unmittelbar Einfluss auf Gesundheit und Wohl dieser Unglücklichen. Es ist ein köstlicher und wahrhaft menschenfreundlicher Grundsatz, Neigungen und Fähigkeiten des Gefangenen bei der Wahl seiner Beschäftigung obwalten zu lassen." Schwierig ist es, die jeweils bestgeeignete Arbeit und ihre Dauer zu ermitteln. Es empfiehlt sich immer, „die Gesetze der Hygiene und nicht die des Eigennutzes zur Grundlage der Arbeitsangelegenheiten in Gefängnissen zu machen".

„Aus dem Gesichtspunkte der polizeilichen Hygiene wird die Frage sich geltend machen, ob man den Strafgefangenen Genüsse erlauben und Gymnastik empfehlen soll.“ „Beischlaf und Gefängnis schließen einander aus. Arbeit und Gymnastik sollen die Hitze des Zeugungstriebes während der Haft dämpfen.“ Genuss des Tabaks in jeder Art soll ausnahmsweise und in beschränktem Ausmaß gewährt werden, um Entziehungserscheinungen von Körper und Gemüt zu vermeiden. Kleine Mengen von Bier und Wein können schwer arbeitenden Gefangenen von Vorteil sein. „Gymnastik und Promenade, und zwar täglich ausgeführt, sind dem Gefangenen ein wahrhaftes Bedürfnis.“

Die Frage, ob gemeinsame oder Einzelhaft der Gesundheit mehr schadet, lässt sich nicht eindeutig beantworten. Erstere ist moralisch schädlich, weil sie den Verhafteten Gelegenheit gibt, sich gegenseitig in den Lastern zu unterrichten. „Die Einzelhaft ist der Gesundheit und dem Leben im allgemeinen weniger vorteilhaft; doch wenn sie gut geleitet wird, wenn der Einzelzellen-Gefangene von Menschenfreunden, vom Arzt, Lehrer, Prediger, Direktor häufig besucht wird, wenn er human behandelt und entsprechend verpflegt wird, dann dürfte die Einzelhaft der Sittlichkeit, Gesundheit und Lebensdauer wohl förderlich sein.“

„Wie bei den Hospitälern so ist auch bei den Gefängnissen die Sterblichkeit der Bewohner ein Maß- stab der Salubrität des Hauses“. Der Vergleich verschiedener Anstalten führt Reich „zu dem Schlusse, dass es unerlässlich sei, alle physisch herabgekommenen, schwächlichen, schlecht genährten Gefangenen durch entsprechende, kräftigende Nahrung und den Einfluss einer umfassenden Hygiene zuvor gesund, stark zu machen. Erst unter dieser Voraussetzung wird der Erziehung Erfolg gesichert, wird die Arbeit zu einem Mittel, Gesundheit und Sittlichkeit zu erhöhen, [sie] überhaupt zu erzeugen.“

„Weil der Verbrecher durch das von der bürgerlichen Gemeinschaft verursachte Elend dazu getrieben wurde, das Gesetz zu verletzen, ist es die Pflicht der Gemeinschaft, in Gefangenenhäusern durch Gewährleistung einer umfassenden Hygiene aus dem Verbrecher einen vollen Menschen, einen gesunden Menschen zu machen, ihn zu bilden, zu veredeln und zu einem nützlichen Mitglied der Gesellschaft zu erziehen.“ Tut sie das nicht, dann ist sie „eine Gesellschaft von Schurken und Vampiren, von gewissenlosen Geizhälsen und Teufeln“ und „verdient den Strick“.

Die Verantwortung des Rates der Wohlfahrt endet nicht mit der Entlassung aus der Haft. Ihm obliegt der Schutz der Entlassenen zumindest bis sich dafür geeignete Vereine gebildet haben, die „unter seinem Auge das Werk der Liebe fortführen“.

\section{Institute der Gewerbetätigkeiten und des Handels}

Dem Rat der Wohltätigkeit obliegt es auch, sich der Institute der Gewerbetätigkeiten und des Handels anzunehmen. Er muss die Besitzer und Verantwortlichen von Fabriken und Werkstätten verpflichten, die „Salubrität der Arbeitsstätten“ zu erhalten. Die Gesundheitspolizei soll dies überwachen und „die Arbeitszeiten feststellen, die Verwendung allzu jugendlicher, schwächlicher, gebrechlicher Menschen zur Fabrikarbeit verbieten und die Arbeitgeber für das Wohl der Arbeitnehmer, so weit überhaupt dies möglich ist, verantwortlich machen".

„Da aber durch dieses von der Hygiene diktierte Verfahren verschiedene wirkliche oder vermeintliche Interessen geschädigt werden, so müssen ökonomische Institute [Maßnahmen], vorzüglich aber muss die Barmherzigkeit [das soziale Verhalten] hier ergänzend wirken, den Schaden gut machen, die schützende Hand über die Jugend, das Alter und die Gebrechlichkeit halten und den Ermatteten neue Kräfte einflößen. Nur durch das Gegengewicht guter, die Sicherheit gewährender Institute, und andererseits der Barmherzigkeit, werden Fabriken und Werkstätten das Wohl der Arbeitenden nicht [schädigend] beeinflussen."

„Die Fabriken sind leider für das zivilisierte Leben unentbehrlich geworden. Es wäre ein großes Glück für die Menschheit, wenn man diese Institute austilgen könnte, ausrotten samt der Gewinnsucht, die sie auch zur Qual für Millionen errichtete. Nun aber fragt es sich, ob das Elend der Fabriken oder jenes des Landes größer sei; ob die Sklaverei des Fabrikproleta- 
riats schwerer wiege als das Joch der Hörigkeit, der Armut mancher Bauernbevölkerungen?" In Erfüllung der Aufgabe als Hygieniker und als „Freund und Verteidiger der geknechteten, ausgesaugten, verachteten armen Teufel, der Fabrikarbeiter und Ackerbauern“, fordert Reich im Namen der Gerechtigkeit und Liebe, „dass die Arbeitenden geschützt werden vor Krankheit und frühzeitigem Tod, vor dem physischen und moralischen Verderben, vor den Übergriffen der Gewinnsucht, der Gewissenlosigkeit und des Egoismus“. „Dieser Schutz wird ihnen zu Teil durch pünktliche Ausführung der Gebote der Hygiene, teils durch die Hand ihrer Arbeitgeber, teils durch ihr eigenes Zutun, teils durch das Wirken des Rates der Wohlfahrt."

Fabriken sollen nicht innerhalb von Städten errichtet werden, sondern, wo es möglich ist, in „Arbeiterstädten“ und zwar so, dass „der Arbeiter zugleich ein Stück eigenes Feld bebauen und einen eigenen Garten pflegen kann“. Wo dies nicht möglich ist und die Fabrik in bewohntem Gebiet errichtet werden soll, so ist es, selbst wenn sie für ihre Arbeiter hygienisch einwandfrei ist, trotzdem möglich, dass ihr Betrieb die Gesundheit von in der Nähe Wohnenden schädigt. Deswegen sind alle Menschen berechtigt, vor der Errichtung einer Fabrik in ihrer Nachbarschaft durch ihre Zustimmung oder Ablehnung die eigenen Gesundheitsinteressen zu wahren.

Die Hygiene einer Fabrik oder einer Werkstätte wird bestimmt durch gute Ventilation, Reinhaltung, schnelle Fortschaffung aller Schädlichkeiten. Die Hygiene des Fabrikarbeiters verlangt „sorgfältige Reinhaltung der Haut, Wechsel der Kleidung, genügende Nahrung, Gymnastik, Exkursionen [Bewegung im Freien], Vorsicht, Mäßigkeit, Sittlichkeit“ und „zeitweiligen Betrieb des Landbaues“. Unter diesen Voraussetzungen wird der Arbeiter gesund bleiben. Ganz besonders kommt es darauf an, den Arbeiter vor den einzelnen Schädlichkeiten, die ihn während der Arbeit treffen können, zu schützen, z.B. vor Schwermetallen und ihren Verbindungen oder Phosphor. Für manche Berufe, z.B. Bergleute, ist es wegen ihrer besonderen Arbeitsbedingungen sehr schwierig, den ihnen in ihrem Beruf begegnenden Gefährdungen zu widerstehen. „Für jede Örtlichkeit treten Modifikationen im Gesundheitsregiment ein, und es ist Sache des Rates der Wohlfahrt, diese Modifikationen zu bestimmen."

Ganz wesentlich ist die Bestimmung der Arbeitsdauer und der Zulassung von Frauen und Kindern zur Fabrikarbeit. Eine auf Dauer zu lange tägliche Arbeitszeit schädigt die Gesundheit. Ihre Verminderung ist aber auch „nötig, um den Arbeiter besser in den Stand zu setzen, geistig sich zu bilden“. „Das Gesetz darf Kinder gar nicht, Frauen nur in beschränktem Maße zur Arbeit zulassen und muss die Zahl der Arbeitsstunden der Hygiene vollkommen gemäß feststellen. In einem Lande wird diese Zahl eine größere sein können, in dem anderen wird sie kleiner sein müssen, je nach der Rasse, der Nahrung und anderer Verhältnisse."
Die tatsächlich gültigen Gesetze über Kinderarbeit sollen die Situation der Familie berücksichtigen. Am besten ist es, wenn der Vater die Sorge für die Familie und seine Kinder selbst tragen kann, weil er einen genügenden Lohn erhält und vielleicht sogar ein eigenes Konto in der Bank seiner Genossenschaft besitzt oder bei Notwendigkeit durch seine Berufsassoziation unterstützt wird. In solchen Fällen mag das Gesetz die Arbeit der Kinder in Fabriken bis zu ihrem sechzehnten Jahr verhindern. Ist der Vater jedoch auf den Lohn des Kindes angewiesen, „dann darf das Gesetz entweder nur sehr wenig fordern oder aber es muss die Gemeinschaft aller Bürger die Kinder der Fabrikarbeiter bis zu deren sechzehnten Jahr ernähren, erziehen und unterrichten".

Zur Tageseinteilung arbeitender Kinder ist es am besten, den Vormittag für die Schule, den Nachmittag für die Arbeit einzuteilen, sodass die Kinder den Tag ausgeruht und geistig aufnahmefähig beginnen können. Da dies nicht immer möglich ist, sind Schulen für diese armen Kinder ohne oder nur von geringem Nutzen. „Fabrikarbeit und Schule schließen einander aus.“ Erst wenn es erreicht sein wird, „dass kein Mensch vor dem zurückgelegten fünfzehnten Lebensjahr die Fabrik oder die Werkstätte als Arbeiter oder Lehrling betreten darf, wird die Schule Nutzen bringen“.

Der Gegensatz von Fabrikarbeitern und in Luxus lebenden Bevölkerungsgruppen wirft soziale Fragen auf. „Der allzu große Luxus ist nicht die Ursache, sondern die Wirkung der Fabriken. Da es nicht in unserer Macht steht, die Fabriken auszutilgen, so sollen wir doch deren fieberhafte Tätigkeit durch Verminderung des Luxus beschränken." Die gänzliche Abschaffung der Fabriken würde aber den Arbeitern die Löhne nehmen und Tausende verhungerten. Hier sieht Reich als einzigen Ausweg, die durch die Beseitigung des übermäßigen Luxus ersparten Summen zum Bau von Arbeiterhäusern, zur Beschaffung leiblicher Bedürfnisse und geistiger Nahrung für die Armen zu verwenden. Es ist also „ein gewisses Maß von Luxus zulässig, ja wünschenswert, solange die Geldwirtschaft noch besteht; es wird dieses bescheidene Maß [von Luxus] die Kinderarbeit und auch die Arbeit während der Nacht nicht erforderlich machen“.

Reich bespricht die speziellen Obliegenheiten der Gesundheitspolizei für einzelne Handwerke und Fabrikarbeiten wie auch der Beschäftigten in Handel und Verkehr.

\section{Militärwesen}

Der Besprechung der Hygiene der Schlachthäuser reiht Reich „die Erörterung über den Krieg und dessen Institute" an und vergleicht beide. Der Mensch wird nicht wie andere Säugetiere des Fleisches wegen geschlachtet, „sondern um ihn im Erdreiche den Würmern als Speise anzubieten, daweilen auf dem Erdboden die Weiber und Kinder der Geschlachteten 
wegen Mangel an Fleisch verkümmern, verschmachten, verhungern“. Die Menschen „begeistern sich für den Krieg, weil die Nächstenliebe nicht in ihren Herzen, sondern nur auf ihren Zungen residiert“. Weil sie noch nicht „vom Viehischen sich emanzipierten, um die ganze Verruchtheit des systematischen Mordens zu begreifen und aus der Tiefe der Überzeugung zu verabscheuen, zu verdammen, zu brandmarken“. „Krieg und Tugend, Krieg und Glückseligkeit, Krieg und Gesundheit" schließen einander aus. Aber es werden die Kriege und damit deren Hygiene und Medizin nicht aufhören, solange Menschen ihre Mitbrüder als für ihren Eigennutz und ihre Blutgier geeignete Objekte halten. „Die beste Hygiene des Krieges ist Tilgung der Selbstsucht, Tilgung der Armut, Hebung der Moral. Die Abschaffung der stehenden Heere ist nur ein palliatives Mittel.“

Da der Krieg sicher noch lange unvermeidlich sein wird, müssen Männer zum Militärdienst. Für ihre Aufnahme in die Nationalgarde oder Volkswehr soll die Konskription [gesetzlich geregelte Einberufung der wehrfähigen Männer], in die Armee das Werbesystem eingesetzt werden. „Durch das Werbesystem werden dem bürgerlichen Leben Kräfte nicht entzogen, es wird aber die Gesellschaft von Individuen befreit, die ihr besondere Vorteile nicht eingebracht hätten, die aber als Krieger ganz am Platze sind.“ „Die Konskription für das stehende Heer ist durchaus unhygienisch“, denn sie führt zum späten Heiraten der Gesündesten und Kräftigsten aus der Bevölkerung. Sie ist aber auch vom „Gesichtspunkt der Moral und der Ökonomie zu verdammen, denn sie wirkt mittelbar entsittlichend und befördert, besonders durch das Moment blutiger Kriege, die Massenarmut.“

Die Sterblichkeit der Soldaten ist selbst im Frieden hoch, auch wenn die Kasernen in trockenen Lagen stehen, aber durch fehlerhaften Bau feucht und „mephitisch“ [Mephitis (lat.) = schädliche Ausdünstung der Erde] sind und Luft und Licht nicht genügenden Zutritt haben. Dazu kommen noch u.a. Unregelmäßigkeiten des alltäglichen Lebens, Entbehrungen, Überanstrengungen und klimatische Schädlichkeiten. Solche Einflüsse und Krankheiten fordern im Krieg mehr Soldatenleben als Feindeinwirkungen. Als Abhilfe sieht Reich „eine umfassende Hygiene, ausgeführt zunächst vom Rat der Wohlfahrt durch die Mittel freier Militärärzte, und alsdann von den Soldaten selbst durch das Mittel hygienischer Bildung und hygienischen Lebens“. Für eine wahre Militärhygiene zur Verhütung der großen Sterblichkeit in den Armeen müssen die Militärärzte selbständig sein, eine in allen medizinischen und hygienischen Dingen entscheidende Stimme haben und vom Rat der Wohlfahrt kräftig unterstützt werden. Tatsächlich verrichten die Militärärzte in den meisten Ländern ihre schwere Aufgabe voll Verantwortung, wofür sie jedoch schlechten Lohn und die Verachtung von Staat und Offizieren erhalten. Somit wäre es am besten, wenn sie nicht zum Soldatenstand gehörten, sondern Organe des Rates der Wohlfahrt wären. Dieser müsste als oberster Leiter der Kasernenverwaltung die alleinige Instanz der Entscheidung über alle hygienischen Entscheidungen und somit auch des Baues der Kasernen sein. Am besten wäre es, die Kasernen niederzureißen und sie, durch ein System kleiner Häuser für je sechs bis acht Soldaten zu ersetzen. Sie könnten aber auch durch Bauten „nach Art der Zellengefängnisse oder Klöster“ ersetzt werden, in denen jedem Soldaten eine Zelle zugewiesen wird.

\section{Soziales Leben}

Bei der sanitären Überwachung von Volksfesten hat die Gesundheitspolizei insbesondere auf die Verhinderung von Stuhlentleerungen in Nähe der Buden und auf die Wahrung der Sittlichkeit durch Entfernung Betrunkener und Verhinderung des Beischlafs auf dem Festplatz zu achten. Aber „einerlei, ob eine Hure in oder außerhalb des Bordells ihr Handwerk treibt: wenn sie gut überwacht, täglich ärztlich untersucht und im Erkrankungsfall sofort in das Hospital gebracht wird, steht für die öffentliche Gesundheit wenig zu besorgen“.

Es ist die Vermeidung der Prostitution überall und in allen Schichten der Bevölkerung anzustreben. Die unbedingte Voraussetzung dafür ist die Beseitigung des Elends. Aussicht auf das allmähliche Erlöschen der Prostitution kommt, wenn die Gesellschaft „auf der Höhe der moralischen Bildung steht“, wenn sie also „von Nächstenliebe und nicht von Selbstsucht regiert wird“. „Moralische Vervollkommnung und Bannung des Elends sind hier die prophylaktischen und heilenden Mittel.“

Die Institution des Leichen- und Begräbniswesen hatte als wichtige Aufgabe, der Furcht der Menschen vor dem Lebendig-begraben-werden entsprechend, Vorkehrungen zur sicheren Feststellung des Todes zu treffen. Dann mussten die Umstände bestimmt werden, unter denen der Tote begraben, verbrannt oder einem Oberflächenwasser übergeben werden soll. Dazu gehörte auch die Regelung von Anlage und Betrieb der Friedhöfe durch die Gesundheitspolizei.

\section{Gesundheitspolizei der Epidemien}

Reich hat seine anfangs der 1870er-Jahre geäußerte Meinung [24-26] über Epidemien in seinen 1894 erschienen „Studien über die epidemischen Krankheiten und deren Verhütung“ [32] gänzlich geändert dargestellt, nämlich wie folgt:

Jedes organisierte Wesen ist ununterbrochen von einer Hülle aus Gasen und Dämpfen umgeben. Strömen diese rasch in den großen Luftkreis, so werden sie zersetzt und bleiben ohne jeden nachteiligen Einfluss auf beseelte Geschöpfe. Je weniger sich eine Persönlichkeit in physischer, moralischer und gesellschaftlicher Beziehung wohl zeigt, desto mehr sind die von ihr ausgeschiedenen Gase und Dämpfe schädlich, 
desto mehr setzt ihr Einfluss das leibliche und seelische Widerstandsvermögen des Individuums selbst und anderer Individuen herab. Wird sodann der Organismus von irgendeiner Schädlichkeit getroffen, so kann er dieselbe nicht überwinden und erkrankt.

„Im Großen und Ganzen fallen Persönlichkeiten mit vollkommen normaler Lebensweise und konzentriertem, die niederen Leidenschaften beherrschendem Seelenleben physischen und psychischen Seuchen nicht zum Opfer.“ Besonders reine, tugendhafte, nüchterne, willensstarke Menschen erkranken nicht während der bösartigsten Seuchen oder durch Berührung von Kranken. Ob man von einer Seuche erfasst wird oder nicht hängt nämlich vom Zustand der Seele ab, „denn alle Widerstandskraft ist Seelenkraft und jedes leibliche Verhältnis ist durch ein seelisches Verhältnis bedingt“. Die Seelenkraft wird vermindert durch heftige Gemütsbewegungen, Ausschweifung in Bauch und Liebe, Einfluss von Elend, Mangel, Lebensnot jeder Art, Aufnahme geistiger Getränke und gesellschaftliche Beziehungen, welche die Seele niederdrücken.

Für die Entstehung einer epidemischen Krankheit ist das Zusammenwirken eines schlechten Zustandes der Seele und eines Genius epidemicus Voraussetzung. Dieser ist eine Gesamtheit von Verhältnissen in jenem Teil des Weltenraumes, den die Erde zu den betreffenden Zeiten durchfliegt, und dazu kommen noch Verhältnisse der Sonne, des Mondes, der Planeten, innere Vorgänge der Erde selbst und Vorgänge in den diese bewohnenden Organismen. Der Genius epidemicus ist also „keine Entität, sondern eine große Gesamtheit von kosmischen und tellurischen, physischen und magischen Umständen und Beziehungen“.

Bei der Kombination von epidemischem Genius und kranker Seele hat Letztere weniger Einfluss auf die „Zellen-Staaten gewisser Gewebe und Flüssigkeiten des Körpers“ und es werden diese in Mikroorganismen umgewandelt. Unter diesen Umständen können sich auch Mikroorganismen der einfachsten Art außerhalb des Körpers bilden und in diesen eindringen. Der ganze Organismus wehrt sich durch Aufgebot der Kraft der Seele, die sich mit Hilfe von Chemie und Physik gegen die Wirkungen des epidemischen Genius richtet. Aber eine gesundheitswidrige Gesamtlebensweise setzt die Seelenkraft herunter und vermindert dadurch das Widerstandsvermögen des Organismus.

Zur Verhütung und Bekämpfung der Entstehung einer epidemischen Krankheit muss man, da die Entwicklung des Genius epidemicus nicht verhindern werden kann, die Entwicklung der höheren Seelenkräfte, der Ästhetik und Moral und die Tilgung der brutalen Leidenschaften durch umfassende Gesundheitspflege des Körpers, der Seele und auch der Gesellschaft erreichen.

Zur Heilung epidemischer Krankheiten ist es notwendig, dem Kranken das höchste Maß von psychischen Kräften zu sichern, um die Seele von den Hindernissen ihrer freien Wirksamkeit zu entlasten. Dies geschieht „durch unmittelbaren psychischen und magischen Einfluss des Arztes, des Priesters, des Pflegers, der Freunde" des Kranken sowie durch dessen eigene Willenskraft und die gesamte körperliche Hygiene. Es ist ein Irrglaube, es könne durch Arzneien eine epidemische Krankheit kuriert werden, denn diese für den Körper fremden Stoffe veranlassen unnütze Abwehrreaktionen und damit schädlichen Kraftaufwand.

Epidemische Krankheiten können Seuchen hervorbringen. Diese entstehen aus den „eigentümlichen Verhältnissen der Welt außerhalb des Menschen“ und vorzüglich durch diesen selbst, nämlich durch „Vorurteil, Unkenntnis, Selbst- und Habsucht, ungeeignete Sparsamkeit und Lieblosigkeit“. Diese sind die „Wirkungen des leiblichen und sittlichen Elends“. Denn „aus dem Elend fließt Vernachlässigung der Gesundheitspflege“ und auch „Übermut und Üppigkeit“ als „Disposition zu den seuchenartigen Leiden“. Die Ausbreitung einer Seuche wird, wie man von der Cholera weiß, nur gehemmt durch eine Lebensart nach den Regeln der Hygiene, also durch Seelenkraft, welche die Fantasie meistert und die Zellen-Staaten in der Bildung von Mikroorganismen psychisch und magisch beherrscht. Daraus geht hervor, wie wertlos Desinfektion, Impfen, Medikamente, Grenzsperren oder Quarantänen sind.

Selbst wenn durch irgendwelche chemische oder physikalische Desinfektionsmaßnahmen alle Mikroorganismen abgetötet würden, so wäre die Seuche noch nicht aus der Welt geschafft. Die Verhältnisse, welche die Mikroorganismen entstehen ließen, wären dadurch nicht im Geringsten verändert. Sie würden ununterbrochen die epidemische Krankheit erzeugen, so lange „seelen-unkräftige“ Individuen da sind, deren Seelenkraft durch schlechte hygienische Verhältnisse geschwächt wird, oder bis anderen epidemische Konstellationen auftreten. Impfungen sind deswegen ungeeignet, weil die in den Körper einverleibten Stoffe dort einen Kampf auslösen und dieser nimmt dem Körper und der Seele Kraft.

Jene edlen Menschenfreunde, die ihre finanziellen Mittel „dazu anwenden, ihren Arbeitern gesundheitsgemäße Wohnungen zu bauen, die Bildung und Veredelung ihnen sichern“, haben den Weg zur Vorbeugung von Epidemien gezeigt. „Sie erziehen massige, reinliche, gebildete, gesunde Menschen, die gute Luft atmen, gutes Wasser trinken, wohl sich kleiden und angemessen wohnen, vor Schädlichkeiten sich zu schützen und in Augenblicken der Gefahr sich zu helfen wissen".

Das Ende der epidemischen Krankheiten ist erst dann zu erwarten, wenn die epidemischen Konstellationen auf körperlich und seelisch gesundheitsgemäß lebende Menschen treffen werden, also „wenn Ungesundheit, Gesundheitswidrigkeit, Hab- und niedere Genusssucht gebannt sind“.

„So wie das Elend die somatischen Seuchen verschuldet, so verursachen Dummheit und Aberglaube die psychischen Seuchen“. Deren Verhütung verlangt 
„Erziehung zu Vernunft und Nächstenliebe, geeignetes diätetisches Verhalten und Vorsicht, sittliche Reinheit, Desinfektion fanatischer Lehren, Lüftung der Räume, die durch das Gift der Selbstsucht, der Lieblosigkeit, der Herzens-Härtigkeit, des praktischen Materialismus, der Unduldsamkeit und Verfolgungssucht verpestet sind“.

Reich fasst zusammen, dass die Volkskrankheiten aus Fehlern des Menschen oder aus besonderen Verhältnissen der Umwelt entstehen. Zur Bekämpfung und Vernichtung der Epidemien gehört also zweierlei: die Verbesserung von uns selbst und die Veränderung der Außenwelt in einer unserer Wohlfahrt entsprechenden Weise. Dafür gibt es nur ein einziges Rezept: „die Hygiene - zunächst die polizeiliche, in zweiter Linie die diätetische, soziale und moralische Hygiene. Sie ist das Zeichen, in dem der Mensch über die Epidemien zu siegen vermag.“

\section{Zusammenfassung}

Entgegen der in der zweiten Hälfte des 19. Jahrhunderts steigenden Bedeutung der sich entwickelnden Bakteriologie und der Kenntnis umweltbedingter chemischer und physikalischer Noxen sieht Eduard Reich als Hauptgrund für die Entscheidung zwischen Gesundheit und Krankheit die Funktionen der „Seele“. Davon seien für das normale Leben von Bedeutung: „Tugend“ (das ist Gemeingeist, Pflichterfüllung, Selbsterkenntnis), Vernunft zum Erkennen der $\mathrm{Zu}$ sammenhänge, Sittlichkeit sowie Nächstenliebe und „Barmherzigkeit“ (soziales Verhalten). Das Ziel der Hygiene sei somit, das Gute zu fördern und das Böse zu verhindern. Sie bewirkt damit Gesundheit, Tugend und Glück, einerseits für den ganzen Menschen oder einzelne seiner Organe andererseits auch für die Gesellschaft. Das Endziel der Hygiene ist für Reich aber nicht bloß die Erhöhung der Leistungsfähigkeit der Bevölkerung für irgendwelche ökonomische oder militärische Zwecke, sondern auch die Gesunderhaltung der Seele.

Entsprechend den Aufgaben und Zielen der Hygiene teilt Reich diese in vier Gebiete.

Die Moralische Hygiene will mit Hilfe von Erziehung, Unterricht und Religion die durch das Gehirn geleiteten Handlungen, Gemütszustände und Leidenschaften für den Normalzustand regulieren.

Die Soziale Hygiene bemüht sich um das Wohl der ganzen Bevölkerung. Das betraf zu Reichs Zeit besonders Fragen der Arbeit, als deren Grundpfeiler er Moral, Hygiene und Ökonomie erkannt und bearbeitet hat, und damals wie heute auch die Folgen von Migrationen.

Die Diätetische Hygiene, wohl die historisch älteste Aufgabe der Gesundheitserhaltung, kümmert sich um die leiblichen Bedürfnisse des Menschen und den Gebrauch seiner Organe. Hierfür sind Vernunft und Vorsicht, Übung und Mäßigung, also Moral und körperliche Ertüchtigung Voraussetzung. Das Ziel ist nicht nur die Erhaltung der Gesundheit, sondern auch die Erreichung eines hohen Alters in Gesundheit.

Die Polizeiliche Hygiene (Gesundheitspolizei) hat durch Erlass und Sicherung der Einhaltung von Maßnahmen für die Gesundheit des Volkes zu sorgen. Sie muss mit Barmherzigkeit und Menschenfreundlichkeit vorgehen und, da das Elend ihr ärgster Gegner ist, die falschen Ansichten einer gefühllosen Ökonomie paralysieren. Die Aufgaben der Gesundheitspolizei der einzelnen Verwaltungsregionen sollen Wohlfahrtsräte mit je einem gesetzgebenden und einem vollziehenden Teil übernehmen, und zwar je ein Wohlfahrtsrat für Gesundheit, für Erziehung und für Sicherheit.

Reichs Auffassung von der Hygiene als Ergebnis der Anwendung von Moral und Nächstenliebe im Leben sowohl jedes Einzelnen als auch der ganzen Gesellschaft rechtfertigt deren wieder vergessene Bezeichnung als „Kultur-Hygiene“.

Interessenkonflikt H. Flamm gibt an, dass kein Interessenkonflikt besteht.

Open Access Dieser Artikel wird unter der Creative Commons Namensnennung 4.0 International Lizenz (http:// creativecommons.org/licenses/by/4.0/deed.de) veröffentlicht, welche die Nutzung, Vervielfältigung, Bearbeitung, Verbreitung und Wiedergabe in jeglichem Medium und Format erlaubt, sofern Sie den/die ursprünglichen Autor(en) und die Quelle ordnungsgemäß nennen, einen Link zur Creative Commons Lizenz beifügen und angeben, ob Änderungen vorgenommen wurden.

\section{Literatur}

\section{Verwendete Literatur}

1. Reich E. Über den Inhalt und die Bedeutung der Hygieine. Wochenbl D Kk Gesd Ärzte Wien. 1870;26:333-5. S. 340-4, 345-7.

2. Reich E. Die Geschichte der Seele, die Hygieine des Geisteslebens und die Civilisation. Minden:J.C. C. Bruns; 1884.

3. Fischer A. Kulturhygienische Ausstellungen. Sozialhyg Mitteil. 1925;3:74-8.

4. Fischer A. Zum 100. Geburtstage des Kulturhygienikers Eduard Reich. Münch MedWochenschr. 1936;83:804-5.

5. Reich E. Vorrede zum zweiten Bande. In: Reich E, Hrsg. Medicinische Abhandlungen für die Gebildeten aller Stände. Bd.2. Würzburg:A.Stuber; 1874.S.3-32.

6. Reich E. Aus meinem Leben. Gotha: Stollbergsche Verlagsbuchhandlung; 1864.

7. Röder J. Eduard Maria Reich (Ahnentafel). Sudetendeutsche Fam. 1932;33(5):129-30.

8. Karbe K-H. Eduard Reich (1836-1919) und sein Wirken für die ,gesamteHygiene"in derGothaer Schaffensperiodevon 1861 bis 1869. Beitr Hochschul- UWissenschaftsgeschichte Erfurts. 1987;88(21):243-56.

9. Reich E. Lehrbuch der Chemie für Studirende und praktische Ärzte. Mit Berücksichtigung der österreichischen und preussischen Pharmakopoe.Erlangen:Enke; 1858.2Bände.

10. Reich E. Lehrbuch der Allgemeinen Aetiologie und Hygieine. Erlangen: Enke; 1858.

11. HirschA. BiographischesLexikonderhervorragendenÄrzte aller Zeiten und Völker. 3. Aufl. Bd. 5. München, Berlin: Urban \& Schwarzenberg; 1962.S. 789-90.

12. Reich E. Zur Staatsgesundheitspflege - Ernste Worte an die bürgerliche Gesellschaft. Leipzig: Otto Wigand; 1861. 
13. Reich E. Die Nahrungs- und Genussmittelkunde, historisch, naturwissenschaftlich und hygieinisch begründet. Göttingen:Vandenhoek\&Ruprecht; 1861.3 Bände.

14. Archiv der Universität Bern. Habilitationsakt „Med. Fakultät, venialegendi Dr. Reich“, Nr. 798, 1860.

15. Das General-Direktorium. Manifest an die deutsche Nation! Im Akt: Vorträge der Justiz und Polizei, Vortrag vom 21.5.1861, Fall 746. Staatsarchiv, Kanton Bern, Sign.: StAB BB IX 420 .

16. Polizei. Bundesbl. d. schweiz. Eidgenossenschaft. Staatsarchiv, Kanton Bern, 1862; Bd. II: 265. Sign.: StAB BB IX 420.

17. Degener H. (ed). Reich Eduard. Wer ist's? Unsere Zeitgenossen. 1909;4:339.

18. Reich E. Geschichte, Natur- und Gesundheits-Lehre des ehelichen Lebens. Cassel:J. C. Krieger; 1864.

19. Die Presse (Wien), 19 (1866), Nr. 53 (24. Febr. 1866), Abendblatt „Kleine Chronik“; Nr. 57 (28. Febr. 1866), Beilage „Aus dem Gerichtssaale“; Nr. 64 (7. März 1866).

20. Flamm H. Die Geschichte der Staatsarzneikunde, Hygiene, Medizinischen Mikrobiologie, Sozialmedizin und Tierseuchenlehre in Österreich und ihrer Vertreter. Wien: Österr. Akademied. Wissenschaften; 2012.

21. Original des Briefes verschollen. Textausschnitte: 1.) Hessler W. Briefwechsel Pettenkofers. Auszüge aus sämtlichen im Archiv des Hygienischen Instituts der Universität München befindlichen Briefen (Auszug S. 47), o.J. \& 2.) Lit. Nr. 4.

22. Reich E. Handschriftlicher Brief an Ernst Haeckel vom 3. August 1871. Ernst-Haeckel-Haus der Friedrich-SchillerUniversitätin Jena. Sign.: ID 20896.

23. Reich E. Volks-Gesundheits-Pflege. Coburg: F. Streit; 1862.

24. Reich E. System der Hygieine. Leipzig: Friedrich Fleischer; 1871.2 Bände.

25. Reich E. Grundriss der Hygieine zum Gebrauche für akademische Vorlesungen und zum Selbstudium. Würzburg: A. Stuber; 1873.

26. Reich E. Die Ursachen der Krankheiten, der physischen und moralischen. 2. Aufl. Leipzig: ErnstFleischer; 1871.

27. Reich E. Die Erblichkeit der Gebrechen des Menschen und dieVerhütung der Gebrechlichkeit. Neuwied, Leipzig: Louis Heuser; 1883.

28. WHO (1946) International Health Conference, New York, Beschluss vom 22.7.1946, in Kraft ab 7.4.1948

29. Ribes F. Traité d'hygiène thérapeutique ou application des moyens de l'hygiène au traitement des maladies. Paris: J. B. Baillière et Fils; 1860.

30. Reich E. Die Gesellschaft als Krankheits-Ursache. Athenaeum. 1876;2:356-9.

31. Reich E. Die Fortpflanzung und Vermehrung des Menschen aus dem Gesichtspunkte der Physiologie und Bevölkerungslehre betrachtet. Jena:Hermann Costenoble; 1880.

32. Reich E. Studien über die epidemischen Krankheiten und deren Verhütung. Leipzig: KarlFr. Pfau; 1894.

\section{Weiterführende Literatur}

33. Reich E. Die Universität in Mähren. Eine Skizze dem mährischen Landtage überreicht. Gotha: Stollberg; 1864.

34. Reich E. Die allgemeine Naturlehre des Menschen. Giessen: EmilRoth; 1865.

35. ReichE. UnsittlichkeitundUnmässigkeitaus demGesichtspunkte der medizinischen, hygieinischen und politischmoralischen Wissenschaften. Neuwied und Leipzig: J. H. Heuser; 1866.

36. Reich E. Die Hygieine und ihr Studium. Erlangen: Enke; 1868.
37. Reich E. Ueber die Entartung des Menschen, ihre Ursachen und Verhütung. Erlangen: Enke; 1868.

38. Reich E. Ueber Unsittlichkeit. Hygieinische und politischmoralische Studien. Neuwied, Leipzig: J.H. Heuser; 1868.

39. Reich E. Ueber den Inhalt und die Bedeutung der Hygieine. WochenblGes ÄrzteWien. 1870;26:333-5. S340-4,345-7.

40. Reich E. Medicinische Abhandlungen für die Gebildeten aller Stände. Würzburg: A. Stuber; 1874. Nur 2 Bände.

41. Reich E. Der Mensch und die Seele. Studien zur physiologischen und philosophischen Anthropologie und zur Physik des täglichen Lebens. Berlin: Fr. Nicolai; 1872.

42. Reich E. Ueber Ursachen und Verhütung der Nervosität und Geistesstörung bei den Frauen. Neuwied, Leipzig: J. H. Heuser; 1872.

43. Reich E. Die Kirche der Menschheit. Neuwied, Leipzig: J. H. Heuser; 1873.

44. ReichE. Studienüber dieFrauen. Jena:Herman Costenoble; 1875.

45. Reich E. Athanaeum. Monatsschrift Anthropologie, Hygieine, Moralstatistik, Bevölkerungs- und Culturwissenschaft, Pädagogik, Höhere Politik, und die Lehre von den Krankheitsursachen. Jena: Hermann Costenoble; 1876. Nur 2 Bände.

46. Reich E. Studien zur Aetiologie der Nervosität bei den Frauen. Neuwied, Leipzig: Louis Heuser; 1877.

47. ReichE. Studienüber dieVolksseele aus dem Gesichtspunkte der Physiologie und Hygieine. Jena: Herman Costenoble; 1879.

48. Reich E. Das Leben des Menschen als Individuum. Die Leibes- und Seelenbeschaffenheit der menschlichen Persönlichkeitund deren Beziehungzu GesundheitundWohlfahrt im gesellschaftlichen Zusammenleben. Berlin: G. Bernstein; 1880.

49. Reich E. Arbeit und Lebensnoth, aus dem Gesichtspunkte der Gesundheitspflege und des Humanismus betrachtet. Berlin: Breitkreuz; 1881.

50. Reich E. Die Emancipation der Frauen, das Elend und die geistige Ueberspannung. Grossenhain, Leipzig: Baumert und Ronge; 1884.

51. Reich E. Der Militär-Arzt mit dem, was darum und daran ist. Essen: A. Silbermann; 1883.

52. Reich E. Die Abhängigkeit der Civilisation von der Persönlichkeit des Menschen, und von der Befriedigung der Lebensbedürfnisse. Minden:J.C.C. Bruns; 1883.

53. Reich E. Studien und Beleuchtungen über Oesterreich. Grossenhain: Baumert und Ronge; 1884.

54. Reich E. Gelehrte, Literaten, gleichwie gelehrte Geschäftsleute. Minden:J.C.C. Bruns; 1885.

55. Reich E. Kritik der Vertheidigung Dr. M. Mackenzie's. Berlin, Leipzig: Louis Heuser; 1888.

56. Reich E. Kalte und Schweiss-Füsse, deren Verhütung und Heilung. Stuttgart:A.Zimmer; 1891.

57. ReichE. DieWahrheitüber dieLungenschwindsucht, deren Entstehung und Verhütung. Berlin:A. Schultze; 1892.

58. Reich E. Geschichte und Gefahren der Fruchtabtreibung. Leipzig: H. Barsdorf; 1906.

59. Reich E. Geschichte und Gefahren der Fruchtabtreibung. Culturgeschichtlich-medicinische Studie. Leipzig: H. Barsdorf; 1898 .

60. Degener HAL. Wer ist's? Zeitgenossen. 1909;4:339.

61. Hinrichsen A. Das literarische Deutschland. 2. Aufl. 1891.

62. HirschA. BiographischesLexikon derhervorragendenÄrzte aller Zeiten und Völker. 3. Aufl. Bd. 4. München, Berlin: Urban \& Schwarzenberg; 1962. S. 750-1.

63. Kürschners Deutscher Gelehrtenkalender. 1913;160-1.

64. v. Wurzbach C. Biographisches Lexikon des Kaiserthums Oesterreich.Bd.25.1873.S. 145-9. 